\title{
Self-Similar Collapse Solutions for Cylindrical Cloud Geometries and Dynamic Equations of State
}

\author{
Lisa Holden, ${ }^{1}$ Kevin Hoppins, ${ }^{1}$, Benjamin Baxter ${ }^{1}$ and Marco Fatuzzo ${ }^{2}$ \\ ${ }^{1}$ Mathematics Department, Northern Kentucky University, Highland Heights, KY, 41099 \\ ${ }^{2}$ Physics Department, Xavier University, Cincinnati, $\mathrm{OH} 45207$ \\ holdenl@nku.edu, fatuzzo@xavier.edu
}

\begin{abstract}
A self-similar formalism for the study of the gravitational collapse of molecular gas provides an important theoretical framework from which to explore the dynamics of star formation. Motivated by the presence of elongated and filamentary structures observed in giant molecular clouds, we build upon the existing body of work on cylindrical self-similar collapse flows by including dynamic equations of state that are different from the effective equation of state that produces the initial density distribution. We focus primarily on the collapse of initial states for which the gas is at rest and everywhere overdense from its corresponding hydrostatic equilibrium profile by a factor $\Lambda$, and apply our results toward the analysis of star formation within dense, elongated molecular cores. An important aspect of this work is the determination of the mass infall rates over a range of the parameters which define the overall state of the gas - the overdensity parameter $\Lambda$, the index $\Gamma$ of the static equation of state, and the index $\gamma$ of the dynamic equation of state. While most of the parameter space explored in this work leads to solutions for which the underlying equations do not become singular, we do include a discussion on how to treat cases for which solutions pass smoothly through the singular surface. In addition, we also present a different class of collapse solutions for the special case $\gamma=1$.
\end{abstract}

Subject headings: hydrodynamics - stars: formation 


\section{INTRODUCTION}

Star formation in our galaxy occurs primarily within giant molecular clouds (GMC) highly nonuniform complexes of molecular gas containing a total mass of $\sim 10^{5} M_{\odot}$ within a radius of $\sim 20 \mathrm{pc}$. These complexes have hierarchical structure that can be characterized in terms of clumps and dense cores surrounded by an interclump gas of density $\sim 5-25 \mathrm{~cm}^{-3}$. Clumps have characteristic densities of $\sim 10^{3} \mathrm{~cm}^{-3}$ and radii ranging between $0.2-2 \mathrm{pc}$, the largest of which are comprised of as many as $\sim 1000$ small $(R \sim 0.1-0.2 \mathrm{pc})$, dense $\left(\sim 10^{4}-10^{5} \mathrm{~cm}^{-3}\right)$ cores whose mass function has been measured to range from $\sim 1-100 M_{\odot}$ (with a peak $\sim 10 M_{\odot}$ ) by Jijina et al. (1999), and more recently, from $\sim 0.2-20 M_{\odot}$ (with a characteristic mass of $\approx 2 M_{\odot}$ ) by Lada et al. (2008). It is the gravitational collapse of these cores within a clump that results in the formation of stars. The dynamics of core collapse is therefore a fundamental component of the star formation process.

The study of self-similar collapse flows has provided an important cornerstone of the current theory of star formation (e.g., Shu et al. 1987), with numerous works appearing in the literature. The original self-similar collapse calculations (Larson 1969ab; Penston 1969ab; Shu 1977) considered isothermal spherical flows. Since then, many generalizations of the collapse have been made. The leading order effects of rotation have been studied, both for the inner pressure free region (Ulrich 1976; Cassen \& Moosman 1981) and for the entire core (Terebey, Shu, \& Cassen 1984). The leading order effects of magnetic fields have also been included (Galli \& Shu 1993ab; Li \& Shu 1996, 1997). More recently, the collapse of magnetized singular isothermal toroids has been studied (Allen, Shu, \& Li 2003; Allen, Li, \& Shu 2003).

While much of the focus has been given to spherically symmetric flows, filamentary and elongated structures in molecular clouds are commonly observed (e.g., Houlahan \& Scalo 1992; Harjunpaa et al. 1999; Jijina 1999), and based on the results of numerical simulations (e.g., Curry 2000; Jappsen et al 2005), appear to be an important aspect of the star formation process. A complete understanding of molecular cloud dynamics thus requires the inclusion of cylindrical geometries. Indeed, several authors have applied self-similar techniques toward the study of how cylindrical structures collapse (Inutsuka \& Miyama 1992; Kawachi \& Hanawa 1998; Hennebelle 2003; Tilley \& Pudritz 2003; Shadmehri 2005). A central element of these studies involves the form of the equation of state used. While an isothermal equation of state clearly provides a reasonable and important starting point for the general study of core collapse, observational evidence points to softer equations of state. Specifically, non-thermal linewidths $\Delta v$ in molecular cloud cores show a correlation with density of the form $(\Delta v)^{2} \sim \rho^{-\beta}$, with $\beta>0$ (e.g., Larson 1981; Jijina et al. 1999). If one interprets the linewidth $\Delta v$ as the effective transport speed in the medium, then the 
corresponding effective equation of state has the form $P \sim \rho^{\Gamma}$, where $\Gamma=1-\beta$ and hence $0<\Gamma \leq 1$.

The self-similar collapse of filamentary structures with polytropic equations of state has been explored by Kawachi \& Hanawa (1998) and Shadmehri (2005), with the latter work also including the effects of magnetic fields. These works, however, considered static equations of state which imposed global constraints on the pressure and density. It is likely that the physical processes which govern a gas will change as that gas undergoes gravitational collapse. We therefore extend the analyses of previous works on cylindrical flows by considering a dynamic equation of state (Fatuzzo, Adams, \& Myers 2004). Specifically, we consider the case in which the dynamic equation of state for the collapsing gas is different than the effective (static) equation of state that produces the initial equilibrium configuration. Here, the static equation of state (as set by $\Gamma$ ) refers to the pressure law that enforces the initial (pre-collapse) configuration for the gas, whereas the dynamic equation of state (as set by $\gamma$ ) refers to the pressure law that describes how the thermodynamic variables of the gas change as the material is compressed during collapse. This process is governed by the entropy evolution equation (introduced in $§ 2.1$ ), so that specific entropy is conserved along a given streamline. However, since the physics that determines the density profiles of the pre-collapse states can be different from the physics that governs the thermodynamics of the collapse flow, we allow $\gamma \neq \Gamma$. Our analysis therefore allows for a more robust model from which to gain insight into the dynamics of filamentary/elongated molecular structures.

An important aspect of this work is its focus on the mass infall rate $\dot{M}$. Collapse flows are often self-similar and have no characteristic mass scale. However, the mass infall rate is one of the most important physical quantities in the star formation problem, and determines, in part, the total system luminosity and the total column density of the infalling envelope. These quantities, in turn, largely account for the spectral appearance of protostellar objects (e.g., Adams, Lada, \& Shu 1987; Adams 1990) since most of the luminosity is derived from material falling through the gravitational potential well of the star. Although the circumstellar disk stores some of the energy in rotational motion, the system luminosity is (usually) a substantial fraction of the total available luminosity

$$
L_{0} \equiv \frac{G M_{s} \dot{M}}{R_{*}},
$$

where $M_{s}$ is the total mass of the star/disk system, $\dot{M}$ is the mass infall rate, and $R_{*}$ is the stellar radius. The stellar radius, which helps determine the depth of the potential well, is itself a function of the mass infall rate (Stahler, Shu, \& Taam 1980).

The paper is organized as follows. We formulate the collapse problem via self-similar methods for the general case of a collapsing cylindrical cloud of gas in $\S 2$. In $\S 3$, we determine 
the range of parameter space that yields collapse solutions by considering the limiting cases $v / x \rightarrow 0$ and $x / v \rightarrow 0$, and illustrate how the ensuing collapse is affected by the initial state of the gas. Guided by these results, we explore in $\S 4$ the collapse of initial states that are out of exact hydrostatic equilibrium by being overdense, and apply our results to the collapse of elongated cores in $\S 5$. We consider cases for which solutions go smoothly through the singular surface in $\S 6$, including the $\gamma=1$ case which leads to a different class of collapse solutions. We present our conclusions in $\S 7$.

\section{FORMULATION OF THE COLLAPSE PROBLEM}

\subsection{Basic Governing Equations}

We adopt a cylindrical coordinate system described by the variables $r, z$ and $\phi$. To keep the problem tractable, we assume dependence only in $r$ (i.e., the cylindrical structure is infinite in length), with the self-gravitating gas described locally by its density $\rho(r, t)$, pressure $P(r, t)$ and (radial) velocity $u(r, t)$, and globally by the mass per unit length $M_{L}(r, t)$ contained within a radius $r$. The gravitational collapse of this fluid is governed by conservation of mass

$$
\frac{\partial M_{L}}{\partial t}+u \frac{\partial M_{L}}{\partial r}=0 \quad \text { and } \quad \frac{\partial M_{L}}{\partial r}=2 \pi r \rho
$$

or equivalently, by the equation of continuity,

$$
\frac{\partial \rho}{\partial t}+\frac{1}{r} \frac{\partial}{\partial r}(r \rho u)=0
$$

and the force equation

$$
\frac{\partial u}{\partial t}+u \frac{\partial u}{\partial r}=-\frac{1}{\rho} \frac{\partial P}{\partial r}-\frac{2 G M_{L}}{r} .
$$

To complete the description required for the evolution of the gas to be solved, the pressure must be specified through a choice of the equation of state. For example, the relation $P=s^{2} \rho$ is used to describe an isothermal gas (where $s$ is the sound speed), whereas a polytropic equation of state $P=\mathcal{K} \rho^{\gamma}$ allows for a more general treatment of the problem. Of course, adopting an equation of state represents a simplification to the real system as it embodies the numerous physical processes which govern the true state of the gas - and how they add/remove energy to/from the gas - into one simple relation. It is quite likely that these processes will change significantly during the collapse, so that the equation of state which governs the gas during the initial stages of collapse will almost certainly have a different form from that which governs the collapse during the later stages of the collapse. We therefore introduce a dynamic polytropic equation of state in our formalism which allows 
the relation between pressure and density to evolve during the collapse (e.g., Fatuzzo, Adams \& Myers 2004). Specifically, we assume that entropy is conserved along a streamline, and use the conservation of entropy equation

$$
\left(\frac{\partial}{\partial t}+u \frac{\partial}{\partial r}\right) \log \left[P / \rho^{\gamma}\right]=0,
$$

to follow the evolution of the pressure. We thus refer to $\gamma$ as the index of the dynamic equation of state. It is important to note that equation (5) describes how a given parcel of gas changes its thermodynamic variables along a streamline, therefore allowing for an equation of state which evolves during the collapse. In contrast, relating the pressure to the density through a fixed (static) equation of state (e.g., $P=\mathcal{K} \rho^{\gamma}$ ) implies a global constraint on those variables.

\subsection{The Similarity Transformation}

As shown in $\S 2.1$, the cylindrical collapse problem is represented mathematically by a set of coupled partial differential equations in time $t$ and radial position $r$. In this section, we find a similarity transformation that reduces this set of PDE's to a set of ordinary differential equations in a new similarity variable $x$ which we define below. In particular, we look for a similarity transformation of the general form

$$
\begin{gathered}
x=A t^{a} r, \quad \rho=B t^{b} \alpha(x), \quad M_{L}=C t^{c} m(x), \\
u=D t^{d} v(x), \quad \text { and } \quad P=E t^{e} p(x) .
\end{gathered}
$$

Here, both the coefficients $(A, B, C, D, E)$ and the indices $(a, b, c, d, e)$ are constants. The reduced fluid fields $(\alpha, m, v, p)$ are dimensionless functions of the (single) dimensionless similarity variable $x$. In this work, the time benchmark $t=0$ corresponds to the instant of the onset of collapse.

The general similarity transformation calculation leads to four equations to specify the five indices $a, b, c, d, e$. We leave the constant $a$ arbitrary for the moment and write the rest of the variables in terms of its value, i.e.,

$$
\begin{gathered}
a=a, \quad b=-2, \quad c=-(2 a+2), \\
d=-(a+1), \quad \text { and } \quad e=-2(a+2) .
\end{gathered}
$$

Similarly, for the coefficients we obtain

$$
A=A, \quad B=(2 \pi G)^{-1}, \quad C=\left(A^{2} G\right)^{-1},
$$




$$
D=A^{-1}, \quad \text { and } \quad E=\left(2 \pi G A^{2}\right)^{-1},
$$

where $G$ is the gravitational constant. We thus obtain reduced equations of motion in the form

$$
\begin{gathered}
(a x+v) \frac{d m}{d x}=(2 a+2) m, \\
\frac{d m}{d x}=x \alpha \\
(a x+v) \frac{1}{\alpha} \frac{d \alpha}{d x}+\frac{d v}{d x}=\left(2-\frac{v}{x}\right), \\
(a x+v) \frac{d v}{d x}+\frac{1}{\alpha} \frac{d p}{d x}=-\frac{2 m}{x}+(a+1) v, \\
(a x+v) \frac{d}{d x} \log \left[p / \alpha^{\gamma}\right]=2(2+a-\gamma) .
\end{gathered}
$$

Note that our similarity transformation is not unique - one can always rescale the coefficients $\{A, B, C, D, E\}$ by a set of dimensionless numbers and obtain new equations of motion with different numerical coefficients.

The first two equations of motion can be immediately combined to obtain an expression for the reduced mass $m(x)$, i.e.,

$$
m=\frac{(a x+v)}{(2 a+2)} x \alpha .
$$

Likewise, multiplying equation (9) by the constant

$$
q \equiv \frac{2}{2 a+2}(2+a-\gamma)
$$

and subtracting the product from equation (13) yields a differential equation which can be integrated to obtain an expression for the reduced pressure

$$
p=\mathcal{C}_{1} \alpha^{\gamma} m^{q}=\mathcal{C}_{1} \alpha^{\gamma+q}\left[\frac{(a x+v)}{(2 a+2)} x\right]^{q},
$$

where $\mathcal{C}_{1}$ is a positive integration constant.

Given the solutions for the reduced pressure $p(x)$ and reduced mass $m(x)$, Eqs. (11) and (12) are the relevant equations of motion to determine the remaining unknown functions $\alpha(x)$ and $v(x)$. Using Cramer's rule, we derive an equivalent set of equations

$$
\frac{d \alpha}{d x}=\frac{\mathcal{A}}{\mathcal{D}} \quad \text { and } \quad \frac{d v}{d x}=\frac{\mathcal{V}}{\mathcal{D}}
$$


where

$$
\begin{gathered}
\mathcal{D}=\frac{(a x+v)^{2}}{\alpha}-\frac{\gamma p}{\alpha^{2}} \\
\mathcal{V}=-\frac{(a x+v)^{2}}{1+a}-\frac{2 p(2+a-\gamma)}{\alpha^{2}}+(a x+v)(1+a) \frac{v}{\alpha}-\frac{\gamma p}{\alpha^{2}}\left(2-\frac{v}{x}\right),
\end{gathered}
$$

and

$$
\mathcal{A}=(a x+v)\left(2-\frac{v}{x}\right)+\frac{\alpha(a x+v)}{1+a}+\frac{2 p(2+a-\gamma)}{\alpha(a x+v)}-(1+a) v .
$$

The physical state of the gas is defined through a choice of the parameters $\left(a, \gamma, C_{1}\right)$, and its collapse is described by the solutions to Eqs. (14) - (20) for the specified set of reduced field variables $v_{i}=v\left(x_{i}\right)$ and $\alpha_{i}=\alpha\left(x_{i}\right)$.

It is mathematically possible to consider "complete" solutions that span the entire available range $-\infty<x<\infty$ (first obtained by Hunter 1977; see also Whitworth \& Summers 1985), and as such, span both negative and positive times. However, it is rather unlikely that molecular clouds will evolve toward their centrally condensed initial configurations in a self-similar manner subject to (only) the physics included in these equations of motion. For example, before the onset of collapse (for $t<0$ ), molecular clouds may evolve through the processes of ambipolar diffusion (at least for small mass scales), shocks, turbulent dissipation, cooling flows, condensation instabilities, and cloud-cloud collisions. In addition, the cloud will most likely initiate collapse before a completely self-similar equilibrium state has been attained; the collapse will only become self-similar asymptotically in time (i.e., the selfsimilar collapse solutions of this paper are intermediate asymptotic solutions to the realistic problem of the collapse of a finite cloud with finite central density). As such, the self-similar solutions of this paper for the protostellar collapse phase $(t>0)$ cannot (in general) be extended to the pre-stellar phase $(t<0)$, as they would likely encounter a critical point and become singular. We therefore limit this discussion to solutions with $0<x<\infty$, sometimes called "semi-complete" solutions.

\section{GENERAL SOLUTIONS TO THE COLLAPSE PROBLEM}

In order to determine what set of parameters $\left(a, \gamma, C_{1}\right)$ yield solutions that describe the collapse of a molecular cloud filament, we obtain analytic solutions to the equations of motion in the limit that $t \rightarrow 0$. As these solutions describe the early stages of the collapse, the gas velocity $u$ must be negative and bounded. For a positive value of $a$, this constraint requires that $u=D t^{-(a+1)} v(x)$ not be singular at $t=0$, which in turn requires that $v(x) \propto x^{\beta} \propto t^{\beta a}$, where $\beta \geq(a+1) / a$. Clearly then, the ratio $v / x \rightarrow 0$ as $t \rightarrow 0$ for solutions relevant to our discussion. 
In this limit, Eq. (9) reduces to a form that can be easily integrated, and along with Eqs. (14) and (16), leads to analytic solutions for the reduced density, mass, and pressure of the form

$$
\begin{gathered}
\alpha=\lambda x^{2 / a}, \\
m=\frac{a}{2 a+2} \lambda x^{(2 a+2) / a},
\end{gathered}
$$

and

$$
p=C_{1} \lambda^{-a q}\left[\frac{a}{2 a+2}\right]^{q} \alpha^{2+a},
$$

where $\lambda$ is a positive constant. The reduced velocity is governed by the limiting form of Eq. (12)

$$
a \frac{d v}{d x}-(a+1) \frac{v}{x}=V_{0} x^{2 / a},
$$

where

$$
V_{0}=-2 \lambda\left[\frac{a}{2 a+2}\right]\left[1+C_{1}\left(\frac{2+a}{a}\right)\left(\frac{2 a+2}{a} \lambda^{a}\right)^{(\gamma-1) /(1+a)}\right] .
$$

Eq. (24) yields a power-law form solution

$$
v=V_{0} x^{(2+a) / a},
$$

indicating that collapse solutions can be found for $a>0$ (since $V_{0}<0$ ). However, the real density becomes time-independant and scales with radius as $\rho \propto r^{2 / a}$ in the limit that $x / v \rightarrow 0$. Since the density approaches zero at small radii, solutions for $a>0$ cannot represent the collapse of filamentary like structures (whose initial density profiles are peaked at $r=0$ ), thereby ruling out this range of parameter space in our work.

Interestingly, the ratio $v / x$ also approaches zero as $t \rightarrow 0$ for the case that $a<0$ (since $x \rightarrow \infty)$. The reduced density, mass and pressure given by Eqs. (21) - (23) therefore also describe the state of the gas at the onset of collapse for this case. However, the real density (which still scales as $\rho \propto r^{2 / a}$ ) now becomes singular as $r \rightarrow 0$. Of course, the equations of motion presented in this work are simply mathematical idealizations to the real, physical problem, for which filaments have a finite density core at small radii $r<r_{C}$ and a large but finite outer boundary $r_{\text {out }}$. This "real" system is expected to follow the self-similar solutions for intermediate length-scales. Indeed, previous numerical work (Foster \& Chevalier 1993) indicates that the collapse of an isothermal core approaches the expected self-similar form when the core has $r_{\text {out }} / r_{C}>20$. Likewise, Fatuzzo, Adams \& Myers (2004) found similar results, with cores that have initial inward velocities more readily approaching the self-similar collapse forms. 
For the case that $a$ is negative, additional constraints are provided by the requirement that the reduced mass $m$ remain positive, and that the reduced pressure $p$ increases as $\alpha$ increases. Together, these physical conditions require $-2<a<-1$, as can be clearly seen by the forms of Eqs. (22) and (23). In addition, collapse solutions (for which $V_{0}<0$ ) require that

$$
\lambda>\lambda_{\text {crit }} \equiv\left[\frac{a}{2 a+2}\right]^{1 / a}\left[-C_{1} \frac{(2+a)}{a}\right]^{(a+1) /(a-a \gamma)} .
$$

We note that $\lambda_{\text {crit }}$ is not defined if $\gamma=1$. Indeed, the $\gamma=1$ case yields a unique class of collapse solutions, and will be considered separately in $\S 6.2$.

Since the real pressure is defined in terms of the constant $A$ through the similarity transforms, we can set $p=\alpha^{a+2}$ without loss of generality. That is, for a real pressure

$$
P=\mathcal{K} \rho^{\Gamma}
$$

setting

$$
A=\left[\mathcal{K}(2 \pi G)^{1-\Gamma}\right]^{-1 / 2}
$$

and $\Gamma=(2+a)$ yields the desired form for the reduced equation of state $\left(p=\alpha^{a+2}\right)$, and sets the integration constant

$$
C_{1}=\left[\frac{2 a+2}{a}\right]^{q} \lambda^{a q}
$$

and, in turn,

$$
\lambda_{c r i t}=\left[-\frac{a^{2}}{(2 a+2)(2+a)}\right]^{1 / a}
$$

To obtain full solutions, one must numerically integrate Eqs. (17) - (20) from a specified initial set of values $\alpha_{i}=\alpha\left(x_{i}\right)$ and $v_{i}=v\left(x_{i}\right)$. We note that one cannot set initial conditions which correspond to the onset of collapse since $x \rightarrow \infty$ as $t \rightarrow 0$. As such, initial conditions are set through an arbitrary choice of $x_{i}>>1$ along with a corresponding value of $\alpha_{i}$ for which $\lambda>\lambda_{\text {crit }}$ and a small but negative value of $v_{i}$. Representative solutions are illustrated in Figs. (1) and (2) for the case that $a=-1.25(\Gamma=0.75)$ and $\gamma=0.25$ (with $C_{1}$ given by Eq. [30]), obtained by integrating inward from $x_{i}=5 \times 10^{3}$ and $\alpha_{i}=5.78 \times 10^{-7}$ (for which $\lambda=1.5 \lambda_{\text {crit }}$ ). The solid curves represent the solutions obtained by setting the value of $v_{i}$ equal to its power-law counterpart, as defined by Eq. (26). The dashed lines represent solutions obtained by setting the value of $v_{i}$ equal to $0.1,0.3,3$ and 10 times the power-law value. It is clear from our results that the reduced density is not sensitive to the initial velocity, as all five cases yield values that differed by less than 0.1 percent. In addition, it is clear that the velocity evolves toward its power-law form as given by Eq. (26). 
For completeness, we note that in the limit that $x / v \rightarrow 0$, the reduced mass approaches a constant value $-m \rightarrow m_{0}$. In turn, the reduced pressure takes the form $p \propto \alpha^{\gamma}$. Taken together, the two limiting solutions presented here thus illustrate how the equation of state smoothly transforms from an initial index of $\Gamma=a+2$ (at early times and/or large radii) to a dynamic index $\gamma$ (at late times and/or small radii). This result is clearly illustrated in Fig. 3, which plots $p$ versus $\alpha$ for the solutions presented in Figs. (1) and (2). The five initial velocities yield results that are within 0.5 percent of each other. As can be easily seen from Fig. (3), the equation of state index evolves from a value of $\Gamma=0.75$ (in the lower left corner of the figure) to a value $\gamma=0.25$ (in the upper right corner), with the transition occurring within a narrow region around $x \approx 1$.

Finally, we note that the equations of motion which define our problem may well yield several different classes of solutions - both physical (e.g., wind solutions) and unphysical. However, solutions which pertain to the collapse of filamentary structures in the absence of shocks (but see $\S 6$ ) are all qualitatively similar, and therefore characterized by solutions such as those presented in Figs. (1) - (3).

\section{COLLAPSE OF OVERDENSE STATES}

The analysis presented in the previous section illustrates that collapse solutions of filamentary structures exist when $-2<a<-1$ and $\lambda>\lambda_{\text {crit }}$. It is easy to show that when $\lambda=\lambda_{\text {crit }}$, Eqs. (21) - (23) yield self similar solutions which describe a gas in hydrostatic equilibrium (for which $v=0$ ). These solutions are fully specified by the equation of state index $\Gamma$ (which we use hereafter to specify the state of the gas instead of $a$ ), and reduce to

$$
\begin{gathered}
\alpha_{E}=\left[\Gamma \frac{(2-2 \Gamma)}{(2-\Gamma)^{2}}\right]^{\frac{1}{2-\Gamma}} x^{\frac{-2}{2-\Gamma}}, \\
m_{E}=\left[\Gamma \frac{(2-2 \Gamma)^{\Gamma-1}}{(2-\Gamma)^{\Gamma}}\right]^{\frac{1}{2-\Gamma}} x^{\frac{2-2 \Gamma}{2-\Gamma}},
\end{gathered}
$$

and $p=\alpha^{\Gamma}$ (assuming that $A$ is set through the relation given by Eq. [29]).

In turn, the real density and mass per unit length profiles are given by the time independent expressions

$$
\rho_{E}=\left[\frac{\mathcal{K}}{2 \pi G}\right]^{\frac{1}{2-\Gamma}}\left[\Gamma \frac{(2-2 \Gamma)}{(2-\Gamma)^{2}}\right]^{\frac{1}{2-\Gamma}} r^{\frac{-2}{2-\Gamma}}
$$

and

$$
M_{L, E}=\left[\mathcal{K}(2 \pi G)^{1-\Gamma}\right]^{\frac{1}{2-\Gamma}} G^{-1}\left[\Gamma \frac{(2-2 \Gamma)^{\Gamma-1}}{(2-\Gamma)^{\Gamma}}\right]^{\frac{1}{2-\Gamma}} r^{\frac{2-2 \Gamma}{2-\Gamma}}
$$


In this section, we consider the collapse of gas structures which are initially at rest, but are out of hydrostatic equilibrium by being everywhere overdense by a factor $\Lambda$. As such, the initial gas (at time $t=0$ ) is described by

$$
\alpha_{i}=\Lambda \alpha_{E} \quad \text { and } \quad v_{i}=0
$$

where $\alpha_{E}$ is the corresponding density profile if the gas were in hydrostatic equilibrium. Essentially, this class of solutions correspond to the solutions discussed in $\S 3$, where $\lambda=$ $\Lambda \lambda_{\text {crit }}$, but with the velocity in the $x \rightarrow \infty$ limit given by Eq. (26), as shown below.

We note that hydrostatic equilibrium solutions of the form

$$
\alpha_{E}=\bar{\Lambda} x^{2 / a} \quad \text { and } \quad m_{E}=\bar{\Lambda} \frac{a}{a+2} x^{(2+2 a) / a},
$$

exist when $\gamma=1$. However, since the reduced pressure takes the form $p=C_{1} \alpha m$, the corresponding balance between the pressure gradient and gravity, as described by Eq. (12),

$$
C_{1} \frac{d}{d x}(\alpha m)=-2 \frac{\alpha m}{x},
$$

is then always maintained regardless of the value of $\bar{\Lambda}$. The collapse scenarios considered in this section therefore cannot occur if the dynamic index $\gamma=1$, regardless of the value of the static index $\Gamma$. For completeness, we explore a different class of collapse solutions with $\gamma=1$ in $\S 6.2$.

With an initial (overdense) state defined in terms of $\Gamma$ and $\Lambda$, as per Eqs. (32) and (36), and the dynamic equation of state defined in terms of $\gamma$, the ensuing collapse solutions can then be obtained by numerically integrating Eqs. (17). The adopted relation $p=\alpha^{\Gamma}$ is maintained for the initial (overdense) state by setting

$$
C_{1}=\Lambda^{\frac{(\Gamma-2)(\gamma-\Gamma)}{1-\Gamma}}\left(\frac{2-\Gamma}{\Gamma}\right)^{\frac{\gamma-\Gamma}{1-\Gamma}}
$$

In practice, however, this initial state corresponds to a time $t_{i}=0$, and in turn, an initial value of $x_{i}=\infty$. We therefore first obtain higher-order analytical expressions for the reduced density and velocity from those presented in $\S 3$ via a series expansion of the reduced equations in the limit $x_{0}>>1$. Doing so yields the following values for the reduced density and velocity:

$$
\alpha_{0}=\Lambda \alpha_{E}\left(x_{0}\right)\left[1-\frac{\Gamma}{2-\Gamma} \Delta_{0} x_{0}^{\frac{-2}{2-\Gamma}}\right]
$$

and

$$
v_{0}=-\Delta_{0} x_{0}^{\frac{-\Gamma}{2-\Gamma}}\left[1+\frac{1}{3}\left\{\Lambda \beta_{0}+\frac{2 \Gamma^{3}}{(2-\Gamma)^{2}} \frac{1}{\left(\Lambda \beta_{0}\right)^{1-\Gamma}}-\frac{\Gamma}{2-\Gamma} \Delta_{0}\right\} x_{0}^{\frac{-2}{2-\Gamma}}\right] \text {, }
$$


where

$$
\beta_{0}=\left[\Gamma \frac{(2-2 \Gamma)}{(2-\Gamma)^{2}}\right]^{\frac{1}{2-\Gamma}}
$$

and

$$
\Delta_{0}=\Lambda\left(\frac{2-\Gamma}{1-\Gamma}\right) \beta_{0}\left[1-\Lambda^{-(2-\Gamma)}\right] .
$$

Full solutions can then be easily obtained by numerically integrating inward from $x_{0}$.

We present solutions for the reduced density and velocity in Figs. $4-7$ for an overdensity parameter of $\Lambda=1.5$ and several different sets of $(\gamma, \Gamma)$. The reduced density clearly exhibits the broken power law profile typical of self-similar collapse solutions. We note that the spectral indices which characterize the reduced density solutions are not sensitive to the choice of $\gamma$, and hence have similar forms when $x<<1$. In contrast, these solutions depend sensitively on the value of $\Gamma$, both in terms of shape when $x>>1$ and in overall normalization. Similar behavior is also clearly observed for the reduced velocity.

As noted in the introduction, self-similar collapse flows have no characteristic mass scale. Instead, the collapse flow feeds material onto the central star/disk system at a welldefined mass infall rate $\dot{M}$. In these flows, the infalling material always approaches free-fall conditions on the inside (in the limit $r \rightarrow 0$ ) and the reduced mass determines the size of the infall rate. Specifically, $m(x) \rightarrow m_{0}$ as $x \rightarrow 0$, and in turn, the mass accretion rate per unit length becomes

$$
\dot{M}_{L}=2(1-\Gamma) \mathcal{K}(2 \pi G)^{1-\Gamma} G^{-1} m_{0} t^{1-2 \Gamma} .
$$

Note that if $\Gamma=0.5, \dot{M}_{L}$ is constant in time, whereas softer (stiffer) equations of state result in temporally increasing (decreasing) mass accretion rates. In contrast, the mass accretion rate for spherically symmetric flows is given by the expression

$$
\dot{M}=(4-3 \Gamma) \mathcal{K}^{3 / 2}(4 \pi G)^{3(1-\Gamma) / 2} G^{-1} m_{0} t^{3(1-\Gamma)},
$$

and is constant in time for an initially isothermal gas (Fatuzzo, Adams \& Myers 2004).

We plot the value of $m_{0}$ as a function of $\Gamma$ for $\Lambda=1.5$ and three different values of $\gamma$ in Fig. 8, and as a function of $\Lambda$ for several sets of $(\Gamma, \gamma)$ in Fig. 9 (the data point in this figure at $\Lambda=1.08$ is discussed in $\S 6.1$ ). As expected, larger infall rates occur for initial states that are more overdense. In addition, stiffer static equations of state result in larger mass accretion rates. In contrast, the dynamic equation of state (as defined by $\gamma$ ) has little effect on the value of $m_{0}$.

The insensitivity of the collapse dynamics to the value of the dynamic index $\gamma$ should not be surprising given the inside-out nature of the collapse (as shown explicitly in §5). 
Specifically, the break at $x \approx 1$ exhibited in the density and velocity profiles shown in Figs. $4-7$ denotes the boundary between gas that is in some part supported by pressure $(x>>1)$ and gas that has lost that support and is therefore approaching free-fall $(x<<1)$. In terms of real quantities, this boundary occurs at a radius

$$
r_{B} \approx \frac{1}{A t^{a}}=\mathcal{K}^{1 / 2}(2 \pi G)^{(1-\Gamma) / 2} t^{2-\Gamma},
$$

that moves outward in time. Since the speed at which this boundary moves is governed by the static equation of state, the collapse dynamics therefore depend sensitively on $\Gamma$. In contrast, the dynamic gas pressure has little effect on the collapse dynamics when $x<1$ $\left(r<r_{B}\right)$, and hence, on the overall collapse.

\section{APPLICATION TO ELONGATED CORES}

Dense star-forming cores in molecular clouds are on average slightly elongated, with some cores exhibiting aspect ratios as large as 5 (Jijina et al. 1999). We apply our results to the evolution of fairly elongated cores, making the highly idealized assumption that their collapse can be reasonably approximated by our formalism. In reality, how such cores collapse likely falls within the limiting cases of cylindrical symmetry (explored here) and spherical symmetry (explored in Fatuzzo, Adams \& Myers 2004).

Interpreting the observed non-thermal linewidths $\Delta v$ in molecular cores as the effective transport speed in the medium, one finds

$$
\Delta v=\left[\frac{\partial P}{\partial \rho}\right]^{1 / 2}=[\mathcal{K} \Gamma]^{1 / 2} \rho^{-(1-\Gamma) / 2}
$$

for an assumed polytropic equation of state $P=\mathcal{K} \rho^{\Gamma}$. Adopting the fiducial values $\Gamma=0.5$ and $\Delta v=1 \mathrm{~km} / \mathrm{s}$ for a density of $5,000 \mathrm{~cm}^{-3}$ (e.g., Larson 1981) then yields a value of $\mathcal{K}=2.6 \mathrm{~g}^{1 / 2} \mathrm{~cm}^{1 / 2} \mathrm{~s}^{-2}$, and in turn, density and velocity profiles given by

$$
\begin{gathered}
\rho(r, t)=2.4 \times 10^{-21} \mathrm{~g} \mathrm{~cm}^{-3}\left(\frac{t}{1 \mathrm{Myr}}\right)^{-2} \alpha(x), \\
u(r, t)=2.3 \mathrm{~km} / \mathrm{s}\left(\frac{t}{1 \mathrm{Myr}}\right)^{1 / 2} v(x),
\end{gathered}
$$

where

$$
x=0.42\left(\frac{r}{1 \mathrm{pc}}\right)\left(\frac{t}{1 \mathrm{Myr}}\right)^{-3 / 2}
$$


In Figs. 10 and 11, we plot the density and velocity profiles for the collapse from an initial state defined by $\Lambda=1.5$ and $\Gamma=0.5$, and a dynamics index $\gamma=0.5$. The solid curve in Fig. 10 depicts the initial density profile, and the dotted curves represent the ensuing profiles (from top to bottom) at times $t=10^{4}, 10^{5}$, and $10^{6}$ yrs. Likewise, the dotted curves in Fig. 11 show the velocity profiles (from bottom to top) at times $t=10^{4}, 10^{5}$, and $10^{6}$ yrs. These results clearly illustrate the inside-out nature of the collapse, with gas inside the transition boundary

$$
r_{B} \approx 2.4 \mathrm{pc}\left(\frac{t}{1 \mathrm{Myr}}\right)^{3 / 2}
$$

falling inwardly away from the overlying gas layers.

From the onset of collapse, it takes a time $t_{*} \sim 10^{5}$ yrs for the formation of a young stellar object to occur, and an additional $t_{d} \sim 5 \times 10^{5}$ yrs before stellar outflows disperse the surrounding core material. Thus, our model predicts that cores associated with young stellar objects would be characterized by densities $\sim 5 \times 10^{4} \mathrm{~cm}^{-3}$ and radii $\sim 0.1 \mathrm{pc}$, in good agreement with observations (Jijina et al. 1999). In addition, the mass accretion rate per unit length is

$$
\dot{M}=\mathcal{K}\left(\frac{2 \pi}{G}\right)^{1 / 2} m_{0}=1.2 \times 10^{3} M_{\odot} \mathrm{Myr}^{-1} \mathrm{pc}^{-1} m_{0}
$$

An elongated core with a length of $l=0.5 \mathrm{pc}$ would accrete a mass of $M_{a c c} \approx 10 M_{\odot}$ in a time $t_{*}$ for a slightly overdense initial state ( since $m_{0} \approx 0.15$ in this case, as can be from the solid curve in Fig. 9), and $M_{a c c} \approx 37 M_{\odot}$ for $\Lambda=1.5\left(m_{0}=0.6\right)$. These results are consistent with the low star formation efficiencies of $2-10 \%$ deduced from observations, but seem to favor initial states that are only slightly overdense.

\section{SMOOTH SINGULAR SOLUTIONS}

\subsection{General Formalism}

The solutions presented in $\S 3$ did not cross through the singular surface, defined in the three-variable space $x, \alpha$, and $v$ as the surface on which $\mathcal{D}=0$. While several different forms of solutions can pass through the singular surface (see, e.g., Lou \& Cao 2007), we focus here on those solutions which pass through it smoothly. That is, we consider solutions for which the derivatives $v^{\prime}=d v / d x$ and $\alpha^{\prime}=d \alpha / d x$ exist on the singular surface. A necessary but not sufficient condition for the existence of these solutions is that $\mathcal{V}$ (and hence $\mathcal{A}$ ) also become zero, which occurs along a unique curve (referred to as the critical curve) on the singular surface for each set of values $\left(a, C_{1}, \gamma\right)$. Alternatively, for solutions 
which represent the collapse from an initially static, overdense state for which $p=\alpha^{\Gamma}$, a unique critical curve exists for each set of $(\Lambda, \Gamma, \gamma)$ values. As a matter of illustration, we plot the $\left(v_{c}, x_{c}\right)$ and $\left(\alpha_{c}, x_{c}\right)$ projections of the critical curve in Figure 12 for the parameters $\Lambda=1.5, \Gamma=0.5, \gamma=0.5$, with the upper panel presenting the reduced density profile, and the lower panel presenting the reduced velocity profile (dotted curves). The solid curves represent the solution for the corresponding collapse dynamics, which clearly does not cross the critical curve (or the singular surface).

In order to properly treat solutions which pass through the singular surface smoothly, we obtain analytical solutions through a Taylor series expansion about the critical curve of the form $x=x_{c}+\delta, v=v_{c}+\delta v_{1}$, and $\alpha=\alpha_{c}+\delta \alpha_{1}$, where $\delta<<1$, and $v_{1}$ and $\alpha_{1}$ are the first derivatives in $v$ and $\alpha$ evaluated on the critical curve. Since the system ODE's are second order, only the first two terms in the expansion are required. Values for $v_{1}$ and $\alpha_{1}$ are obtained by substituting these terms into equations (17) and expanding in $\delta$. Doing so then yields the relations

$$
\alpha_{1}=\left(2-\frac{v_{c}}{x_{c}}-v_{1}\right) \frac{\alpha_{c}}{v_{c}-(2-\Gamma) x_{c}},
$$

and

$$
-(1+\gamma) v_{1}^{2}+\left[4 \Gamma-1+2(1-\gamma) \frac{v_{c}}{x_{c}}\right] v_{1}+C_{v}=0,
$$

where

$$
C_{v}=\frac{\alpha_{c}}{1-\Gamma}\left(\Gamma-\frac{v_{c}}{x_{c}}\right)+2\left(\Gamma+1-\frac{\Gamma}{\gamma}\right) \frac{v_{c}}{x_{c}}-\gamma \frac{v_{c}^{2}}{x_{c}^{2}}-\frac{2 \Gamma^{2}}{\gamma} .
$$

The first derivative $v_{1}$ is then the real, positive solution to the above quadratic equation. Full solutions can then be obtained by numerically integrating inward $(\delta<0)$ and outward $(\delta>0)$ from the corresponding Taylor series solutions.

As an example, we consider the collapse from an initial state defined by the parameters $\Lambda=1.08, \Gamma=0.25$ and $\gamma=0.5$. Numerically integrating Eqs. (17) inward from an initial value of $x_{0}=10^{4}$ (as described in $\S 4$ ), one finds that the equations become singular as $x \rightarrow 0.336$, i.e., as the solutions approach the singular surface. Since a real and positive solution $v_{1}$ exists for Eq. (54) at $x_{c}=0.336$ for this case, this solution can thus pass smoothly through the singular surface (by crossing through the critical curve), and can be matched to the Taylor series solution on the other side of the critical curve. Further numerical integration shows that this solution also crosses smoothly through the singular surface at a second point $x_{c}=0.0322$, as illustrated in Figure 13. The solid curve in the upper (lower) panel presents the reduced density (velocity) profile obtained for our solution, and the dotted curves represent the $\left(\alpha_{c}, x_{c}\right)$ and $\left(v_{c}, x_{c}\right)$ projections of the critical curve for the case being considered. It is interesting to note that $\alpha$ approaches $\alpha_{c}$ as $x \rightarrow 0$. The 
value of $m_{0}$ was also determined and is depicted in Fig. 9 by the solid square data point, and is consistent with the extrapolation of the dot-dash curve corresponding to the same $(\Gamma$, $\gamma)$ values.

\subsection{The $\gamma=1$ case}

As noted in $\S 4$, density profiles that are out of hydrostatic equilibrium by being everywhere overdense (i.e., are of the form $\alpha_{i}=\Lambda \alpha_{E}, v_{i}=0$ ) do not exist for the $\gamma=1$ case, regardless of the form for the initial equation of state as defined by $a$ and $C_{1}$. This result is somewhat surprising given the apparent insensitivity that the solutions presented in $\S 4$ have on the choice of $\gamma$, but is borne out through numerical investigation. However, a different class of collapse solutions which passes smoothly through the singular surface does exist for this case, and is presented here for completeness. A full analysis of all solutions with $\gamma=1$ (e.g., wind solutions, shock solutions) is outside the scope of this work and will be presented elsewhere.

To keep our analysis as general as possible, we describe the gas in terms of $a$ and $C_{1}$ throughout this subsection. It is easy to show that when $\gamma=1$, the critical curve on the singular surface becomes a straight line defined by the linear relation $v_{c}=k x_{c}$ and a constant reduced density

$$
\alpha_{c}=\frac{(2+2 a)(a+k)}{C_{1}},
$$

where $k$ is a (real) solution to the quadratic equation

$$
\left[\frac{2}{C_{1}}-1\right] k^{2}+\left[3+\frac{4 a}{C_{1}}\right] k+\left[4 a+2 a^{2}+\frac{2 a^{2}}{C_{1}}\right]=0,
$$

derived by imposing the condition that $\mathcal{D}=\mathcal{V}=\mathcal{A}=0$. Given our focus on collapse solutions, we plot the negative roots of Eq. (57) in Figure 14 as a function of $a$ for values of $C_{1}=1.2,1.5,1.8,2.1$ and 2.4 .

Solutions that pass through the critical curve can be obtained by numerically integrating inward and outward from both sides, using the analytical solutions obtained via the Taylor series expansion presented in $\S 6.1$ (where $v_{c}=k x_{c}$ ). We note that the values of $v_{1}$ and $\alpha_{1}$ obtained by solving Eqs. (43) - (55) are now independent of $x_{c}$ - a consequence of the ODE's

which describe the gas dynamics (Eqs. 17) being invariant to the scaling transformation $x \rightarrow \eta x, v \rightarrow \eta v, \alpha \rightarrow \alpha, p \rightarrow \eta^{2} p$, and $m \rightarrow \eta^{2} m$ for the case $\gamma=1$. We therefore set $x_{c}=1$ in our analysis without loss of generality.

Through numerical exploration, we find a class of solutions that have the limiting form 
$\alpha \propto x^{2 / a}$ and $v \propto x^{(1+a) / a}$ in the limit $x \rightarrow \infty$, and $\alpha \propto x^{-2 / C_{1}}$ and $v \propto x^{\left(2-C_{1}\right) / C_{1}}$ in the limit $x \rightarrow 0$, where $1<C_{1}<2$. To illustrate this type of collapse flow, we plot solutions for the case $a=-1.05$ and $C_{1}=1.5$ in Figure 15. The upper panel presents the reduced density profile, and the lower panel presents the reduced velocity profile. The dotted curves in these panels represent the $\left(\alpha_{c}, x_{c}\right)$ and $\left(v_{c}, x_{c}\right)$ projections of the critical line. Solutions for the real density and real velocity, scaled so that $x=1$ corresponds to $t=1 \mathrm{Myr}$ and $r$ $=1 \mathrm{pc}$, are presented in Figs. 16 and 17 for times 0.01, 0.1, 1 and 10 Myrs. Clearly, this case represents a class of collapse solutions characterized by a nonzero initial inward velocity that slows with time at a fixed radius after the gas becomes isothermal 1 . Indeed, the gas at large radii/early times $(x \rightarrow \infty)$ is moving inward with nearly constant velocity, though the magnitude of the velocity differs from one part of the flow to another (i.e., each "parcel" of gas moves with a nearly constant velocity, such that $d u / d t \approx 0$, but the velocity field for the gas depends on $r$ and $t)$. The density increases as the gas falls inward $(x \rightarrow 0)$, leading to a rise in pressure that eventually becomes effective at slowing the inward collapse. The break in density at $x \sim 1$ in this case results from the slowing down of the infalling gas, rather than from an inward-out collapse associated with typical self-similar collapse solutions. This class of solutions could, in part, idealize the dynamics of a cylindrical structure collapsing after the loss of magnetic support via ambipolar diffusion, where it becomes isothermal as turbulence dissipates during the collapse.

\section{CONCLUSION}

The main goal of our work was to obtain self-similar solutions which describe the collapse of an initially stationary, cylindrically symmetric gas that is overdense from hydrostatic equilibrium by a factor $\Lambda$. Hydrostatic equilibrium profiles are easily found for an assumed static equation of state $p=\alpha^{\Gamma}$, as specified through the choice of the index $\Gamma$. Unlike previous works, we allow the equation of state to evolve during the collapse, under the condition that entropy is conserved along a streamline. Doing so allows the equation of state to change from its initial form (as defined by $\Gamma$ ), to a different polytropic form as defined by the dynamic index $\gamma$. Physical solutions describing this type of collapse require $0<\Gamma<1$ and $\gamma \neq 1$. We present solutions for which the system equations do not become singular, as well as solutions which pass smoothly through the singular surface.

Our solutions clearly exhibit the broken power law profiles typical of self-similar collapse

\footnotetext{
${ }^{1}$ This class of solutions is analogous to the Type II solutions presented in Lou \& Cao (2007) for a spherical geometry with $\gamma=4 / 3$
} 
flows. We find that the spectral indices which characterize the reduced density solutions are not sensitive to the choice of $\gamma$, and hence have similar forms when the similarity variable $x=A t^{a} r<<1$. In contrast, these solutions depend sensitively on the value of $\Gamma$, both in terms of shape when $x>>1$ and in overall normalization. Similar behavior is also clearly observed for the reduced velocity solutions. The insensitivity of the collapse dynamics to the value of $\gamma$ results from the inside-out nature of the collapse. Specifically, the break at $x \approx 1$ exhibited in the density and velocity profiles (as shown in Figs. 4-7) occurs as a result of the gas being in some part supported by pressure $(x>>1)$ evolving to a state approaching freefall $(x<<1)$ as a result of the loss of that pressure support. This break-point also denotes a transition from an initial (static) equation of state $\left(p=\alpha^{\Gamma}\right)$ to a dynamic equation of state $\left(p \propto \alpha^{\gamma}\right)$, with the transition occurring within a narrow region around $x \approx 1$. In terms of real variables, this transition occurs at a boundary which moves outward through the gas as governed by the static equation of state. As such, the gas dynamics depend sensitively on the value of $\Gamma$ (which, of course, also governs the density profile $\rho(r)$ of the initial state). In contrast, the gas pressure within this boundary, as described by the dynamic equation of state (and in turn $\gamma$ ), has little effect on the overall collapse dynamics.

Although self-similar collapse flows have no characteristic mass scale, the collapse flow feeds material onto the central star/disk system at a well-defined mass infall rate $\dot{M}$. Since the infalling material always approaches free-fall conditions on the inside (in the limit $r \rightarrow$ 0 ), the reduced mass determines the size of the infall rate, and is therefore an important parameter in the collapse problem. Our analysis shows that, as expected, larger infall rates occur for initial states that are more overdense. In addition, stiffer static equations of state result in larger mass accretion rates. In contrast, the dynamic equation of state (as defined by $\gamma$ ) has little effect on the value of $m_{0}$.

Our results indicate that collapse from an overdense state initially at rest cannot occur if $\gamma=1$, regardless of the form for the initial equation of state. This result is somewhat surprising given the apparent insensitivity to $\gamma$ exhibited by all other solutions we obtain. However, we do find a different class of collapse solutions which passes smoothly through the singular surface when $\gamma=1$. These solutions are analogous to the Type II solutions presented in Lou \& Cao (2007) for a spherical geometry with $\gamma=4 / 3$.

\section{Acknowledgments}

We thank the anonymous referee for useful comments. We also thank Fred Adams for

many useful discussions. BB was supported by the Greaves Fund at Northern Kentucky University. MF was supported by the Hauck Foundation at Xavier University. 


\section{REFERENCES}

Adams, F. C. 1990, ApJ, 363, 578

Adams, F. C., Lada, C. J., \& Shu, F. H. 1987, ApJ, 321, 788

Allen, A., Li, Z.-Yi., \& Shu, F. H. 2003, ApJ, 599, 363

Allen, A., Shu, F. H., \& Li, Z.-Yi. 2003, ApJ, 599, 351

Cassen, P., \& Moosman, A. 1981, Icarus, 48, 353

Curry, C., \& McKee, C. F. 2000, ApJ, 528, 734

Fatuzzo, M., Adams, F. C., \& Myers, P. C. 2004, ApJ, 615, 813

Foster, P. N., \& Chevalier, R. A. 1993, ApJ, 416, 303

Galli, D., \& Shu, F. H. 1993a, ApJ, 417, 220

Galli, D., \& Shu, F. H. 1993b, ApJ, 417, 243

Hennebelle, P. 2003, A\&A, 397, 381

Harjunpaa, P., Kaas, A. A., Carlqvist, P., \& Gahm, G. F. 1999, A\&A, 349, 912

Houlahan, P., \& Scalo, J. M. 1992, ApJ, 393, 172

Hunter, C. 1977, ApJ, 218, 834

Inustsuka, S., \& Miyama, S. M. 1992, ApJ, 388, 392

Jappsen, A. K., Klessen, R. S., Larson, R. B., Li, Y., \& MacLow, M. M. 2005, A\&A, 435,611

Jijina, J., Myers, P. C., \& Adams, F. C. 1999, ApJ Suppl., 125, 161

Kawachi, T., \& Hanawa, T. 1998, PASJ, 50, 577

Lada, C. J., Muench, A. A., Rathborne, J., Alves, J. F., \& Lombardi, M. 2008, ApJ, 672,410

Larson, R. B. 1969a, MNRAS, 145, 271

Larson, R. B. 1969b, MNRAS, 145, 297

Larson, R. B. 1981, MNRAS, 194, 809

Li, Z.-Y., \& Shu, F. H. 1996, ApJ, 472, 211L 
Li, Z.-Y., \& Shu, F. H. 1997, ApJ, 475, 237

Lou, Y.-Q., \& Cao, Y. 2008, MNRAS, 384, 611

Penston, M. V. 1969a, MNRAS, 144, 425

Penston, M. V. 1969b, MNRAS, 145, 457

Shadmehri, M. 2005, MNRAS, 356, 1429

Shu, F. H. 1977, ApJ, 214, 488

Shu, F. H., Adams, F. C., \& Lizano, S. 1987, A R A \& A, 25, 23

Stahler, S. W., Shu, F. H., \& Taam R. E. 1980, ApJ, 241, 637

Terebey, S., Shu, F. H., \& Cassen, P. 1984, ApJ, 286, 529

Tilley, D. A., \& Pudrit, R. E. 2003, ApJ, 593, 426

Ulrich, R. K. 1976, ApJ, 210, 377

Whitworth, A., \& Summers, D. 1985, MNRAS, 214, 1 


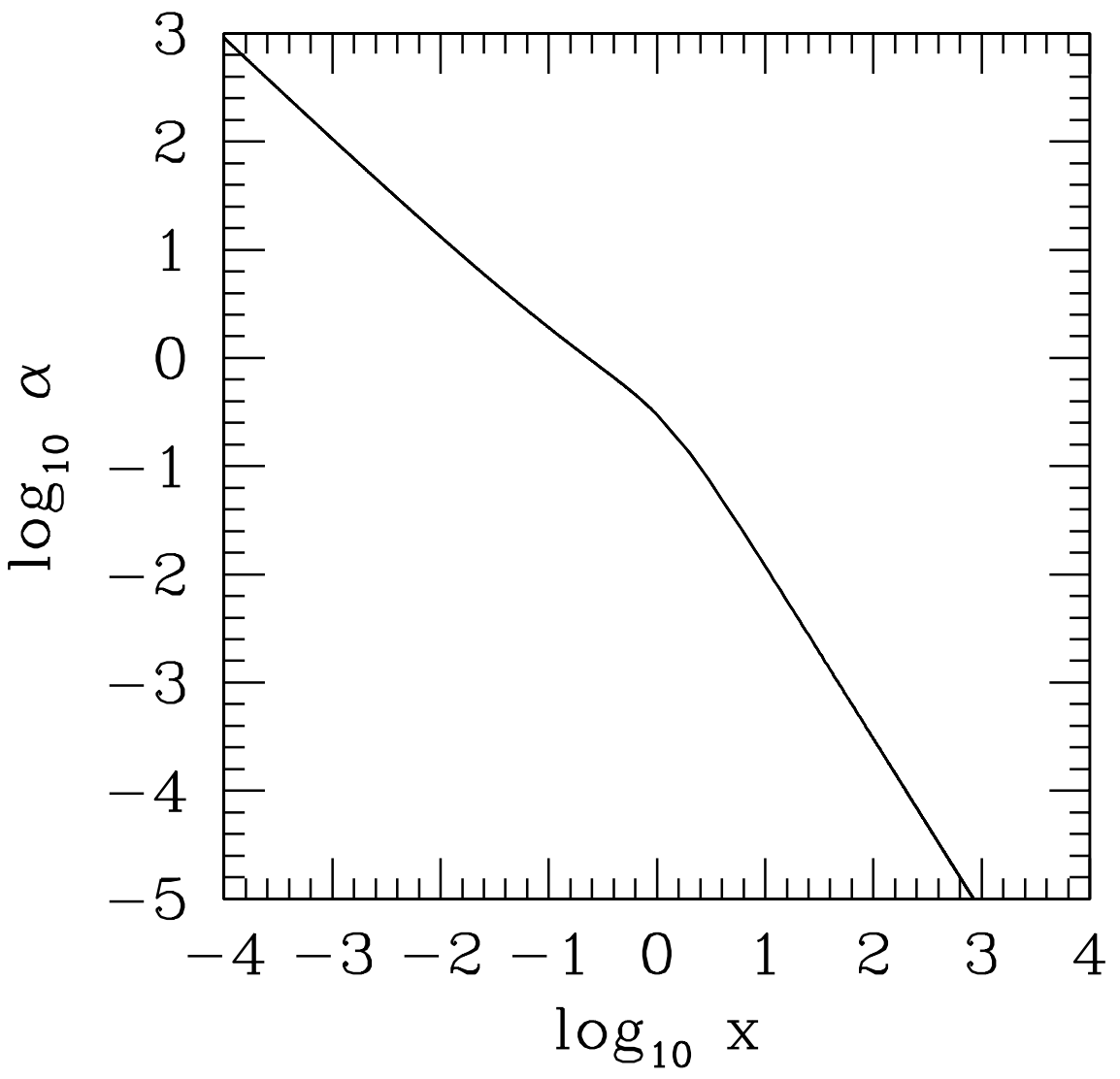

Fig. 1.- Self-similar solutions for the reduced density describing the collapse of a gas defined by the parameters $a=-1.25$ and $\gamma=0.25$. All solutions are integrated inward from an initial set of values $x_{i}=5 \times 10^{3}$ and $\alpha\left(x_{i}\right)=5.78 \times 10^{-7}$, and the five values of $v_{i}$ as discussed in the text. All five solutions are within 0.1 percent of each other, illustrating that the self-similar density profiles are not sensitive to the initial values of the reduced velocity. 


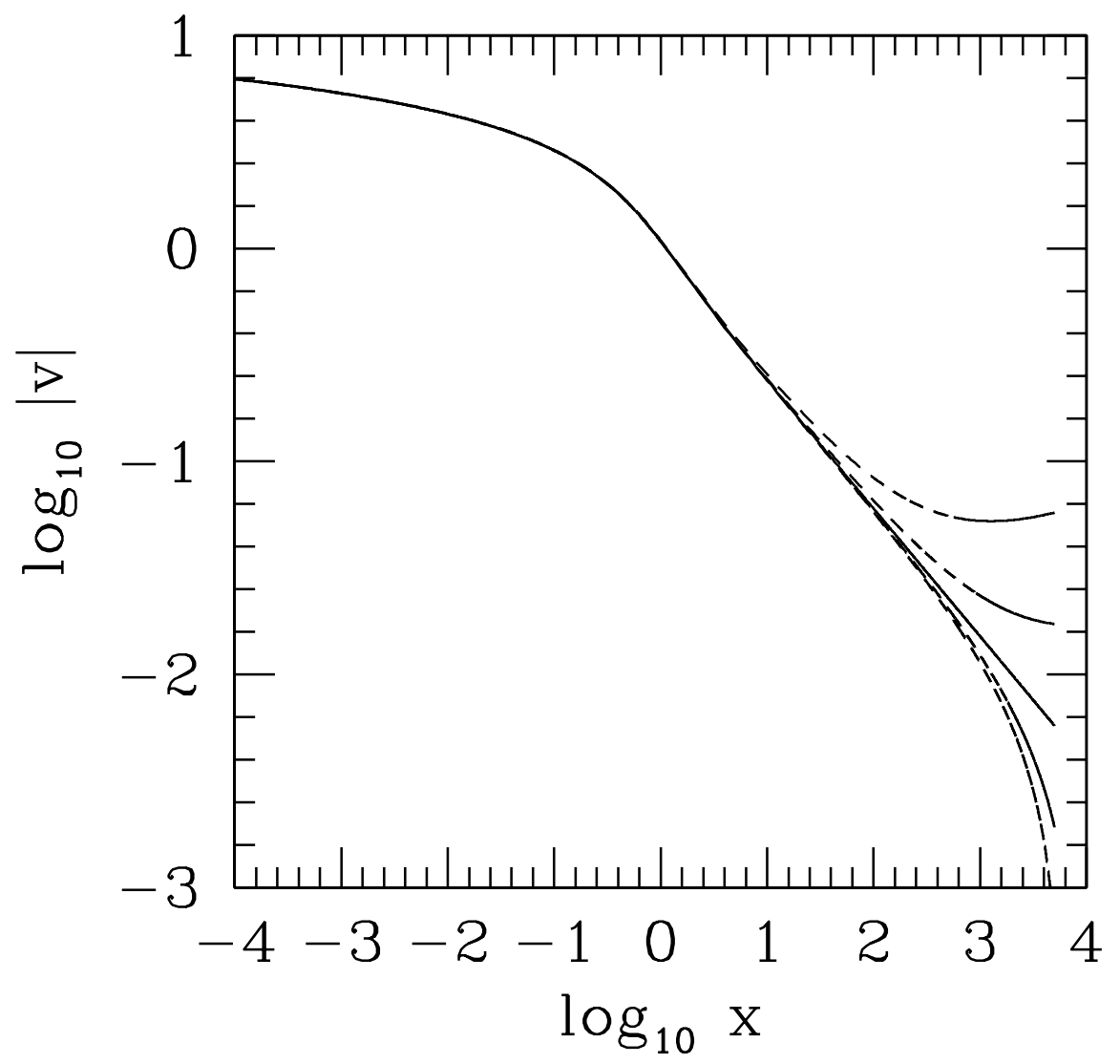

Fig. 2.- Self-similar solutions for the reduced velocity describing the collapse of a gas defined by the parameters $a=-1.25$ and $\gamma=0.25$. All solutions are integrated inward from an initial set of values $x_{i}=5 \times 10^{3}$ and $\alpha\left(x_{i}\right)=5.78 \times 10^{-7}$, and the five values of $v_{i}$ as discussed in the text. 


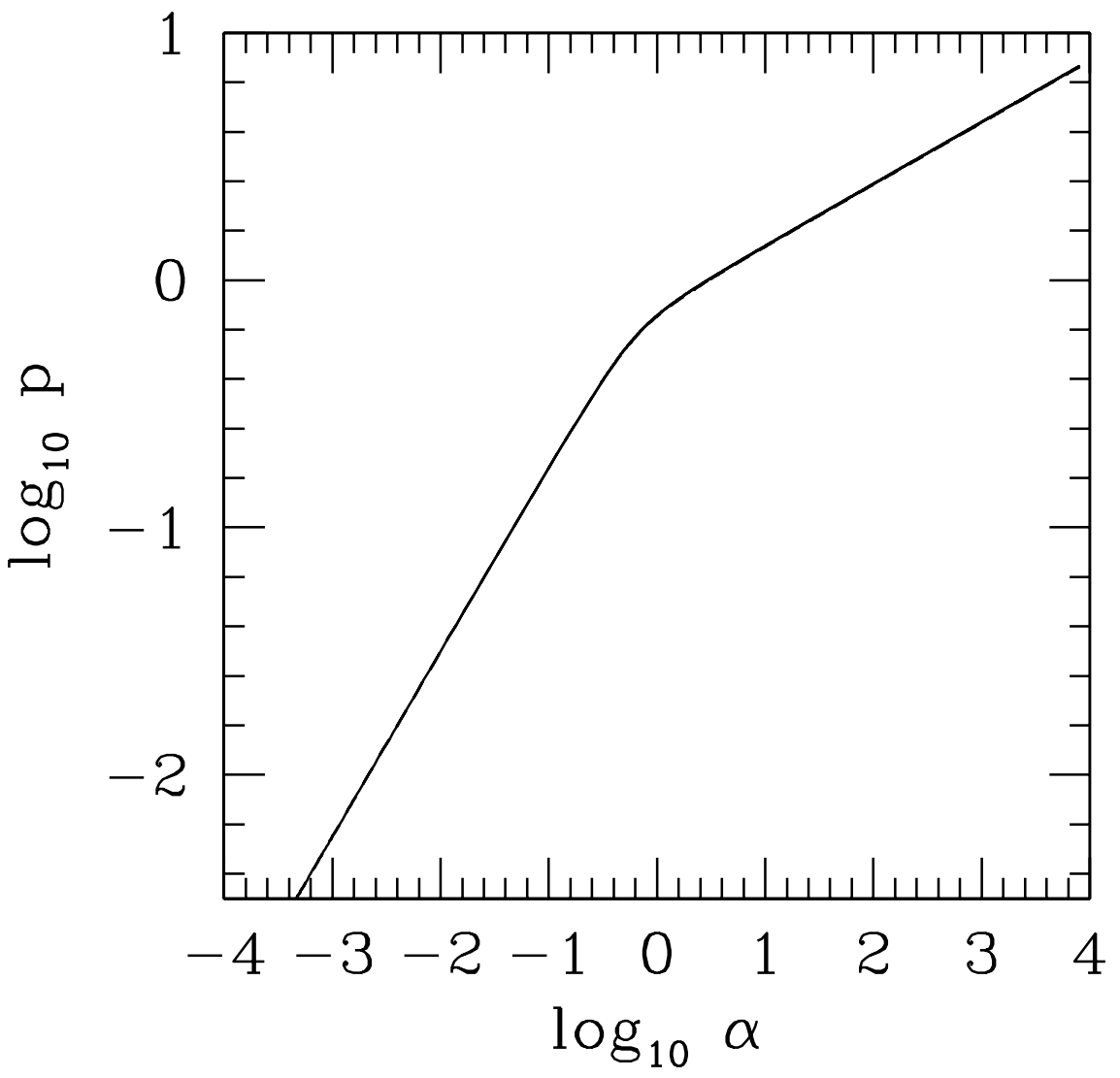

Fig. 3.- $\log p-\log \alpha$ profiles for the solutions presented in Figs. (1) and (2), illustrating the transition during the collapse from a static equation of state $\left(p=\alpha^{a+2}=\alpha^{0.75}\right)$ as shown in the lower left corner to a dynamic equation of state $\left(p \propto \alpha^{\gamma} \propto \alpha^{0.25}\right)$ as shown in the upper right corner. 


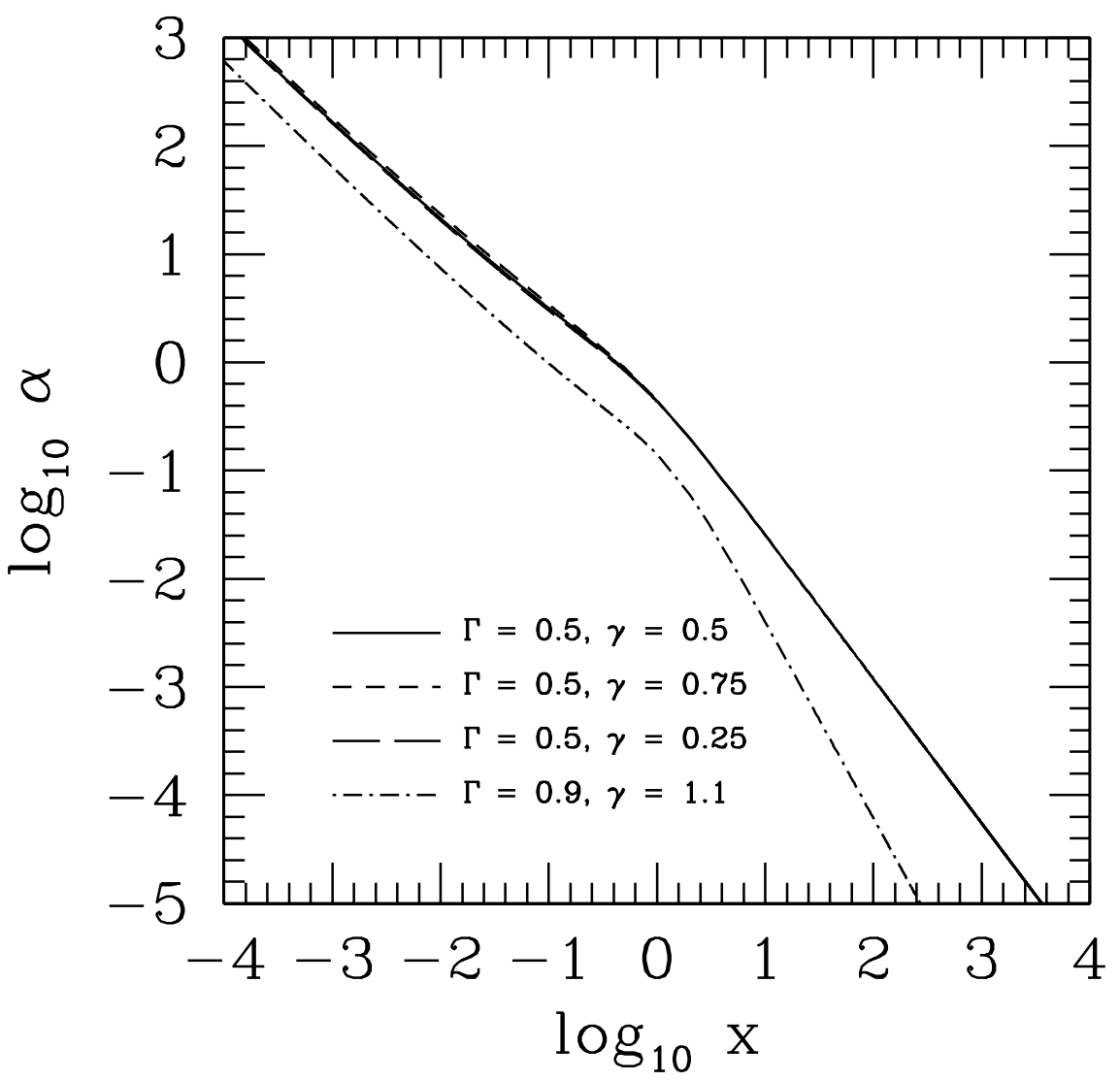

Fig. 4.- Self-similar solutions for the reduced density describing the collapse of an initially static gas overdense from its hydrostatic equilibrium state by a factor of $\Lambda=1.5$. The four profiles correspond to the four different choices of static and dynamics equations of state, as defined by the specified values of $\gamma$ and $\Gamma$. 


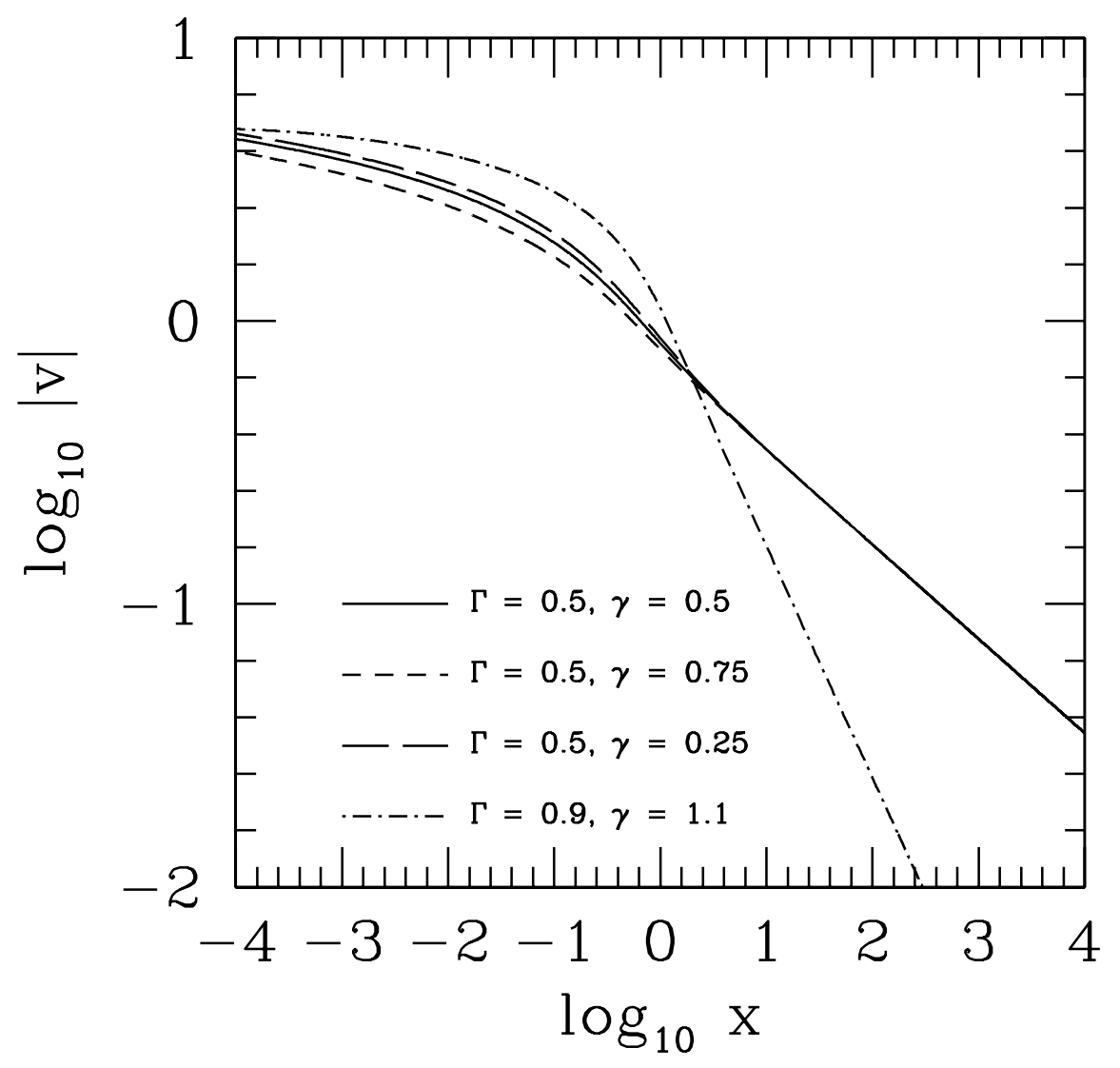

Fig. 5.- Self-similar solutions for the reduced velocity describing the collapse of an initially static gas overdense from its hydrostatic equilibrium state by a factor of $\Lambda=1.5$. The four profiles correspond to the four different choices of static and dynamics equations of state, as defined by the specified values of $\gamma$ and $\Gamma$. 


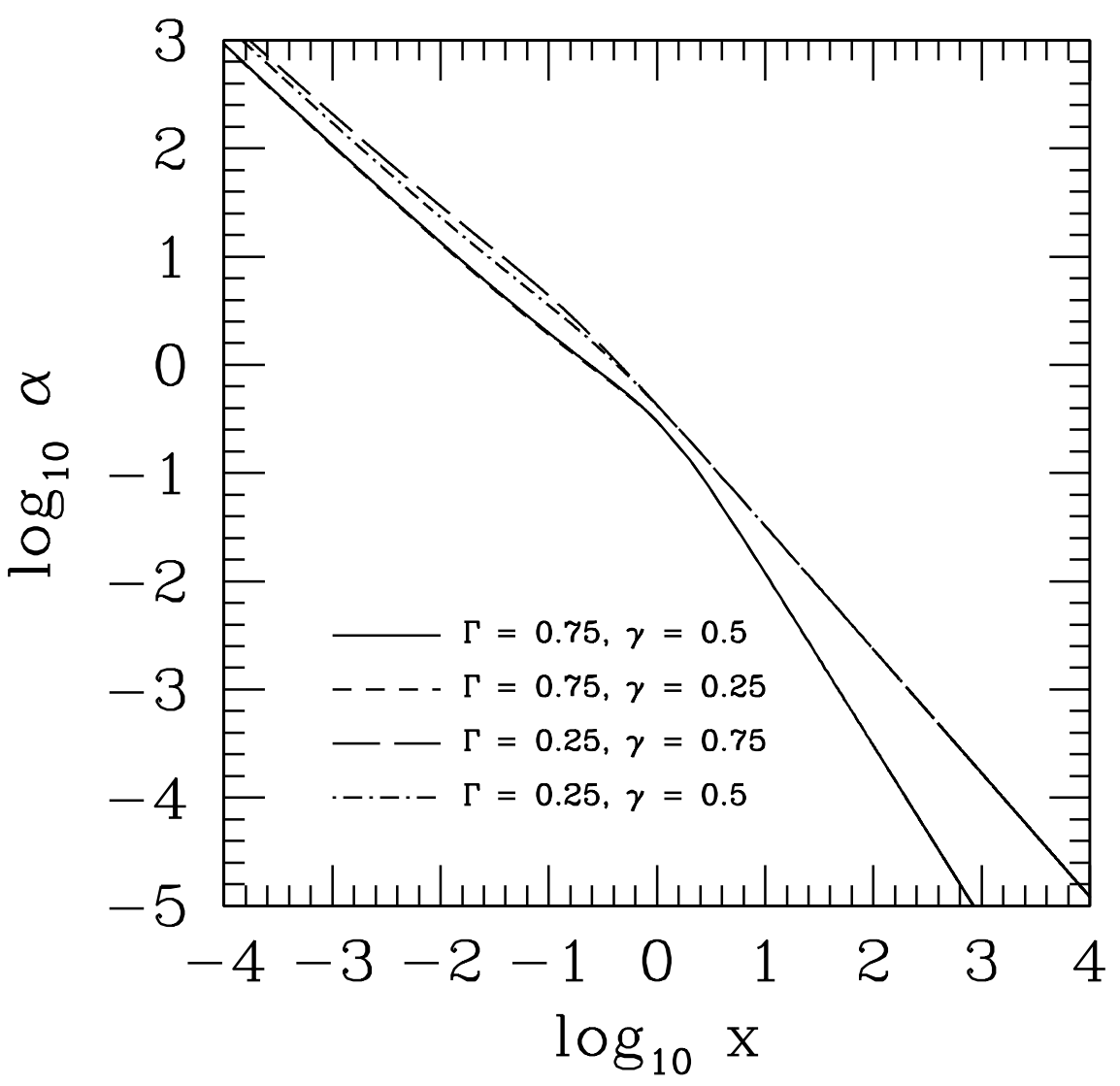

Fig. 6.- Same as Figure 4, but for a different set of values for $\gamma$ and $\Gamma$. We note that the solid curve and short-dashed curve lie over each other in this Figure, and are therefore hard to distinguish. 


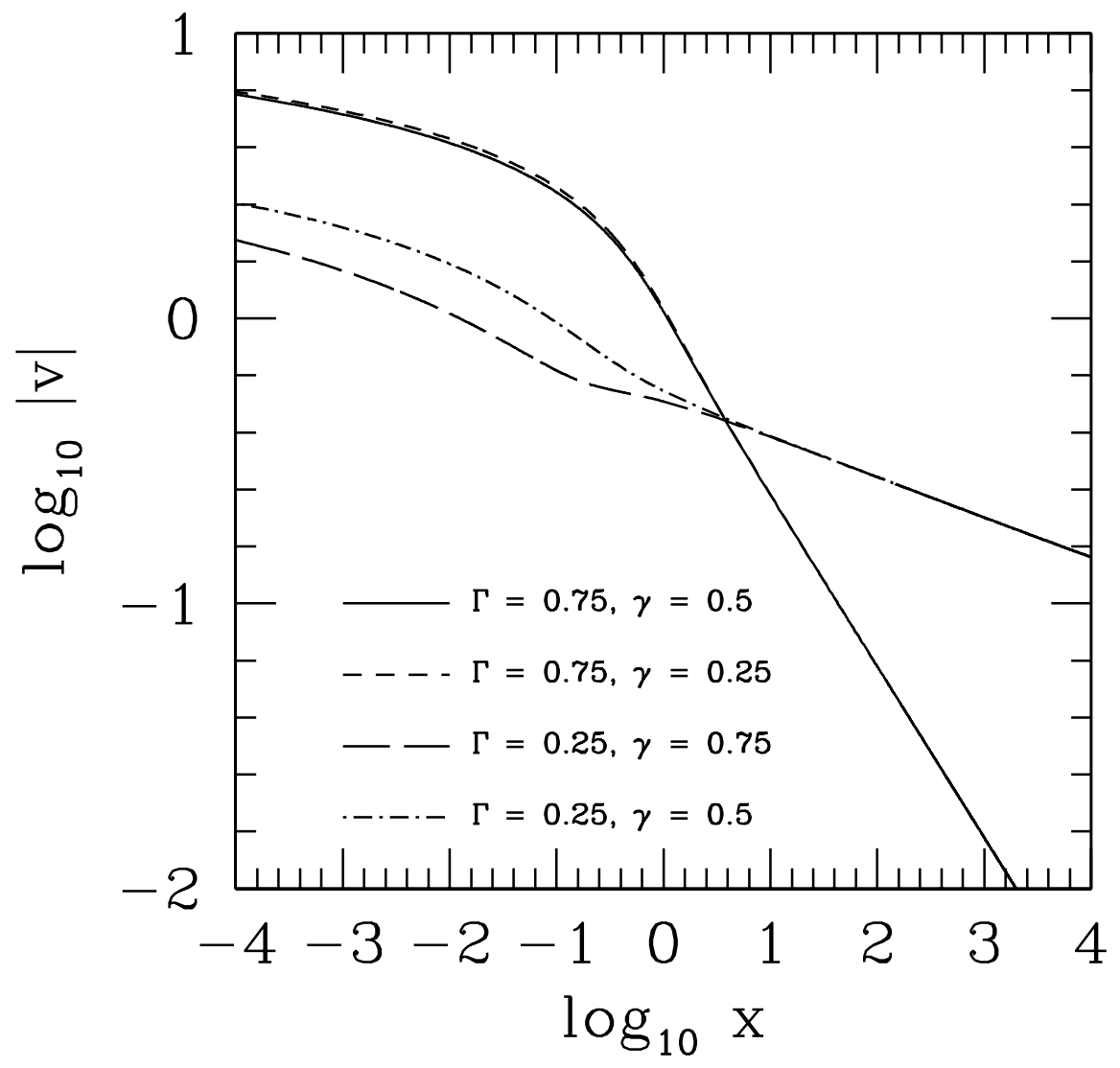

Fig. 7.- Same as Figure 5, but for a different set of values for $\gamma$ and $\Gamma$. 


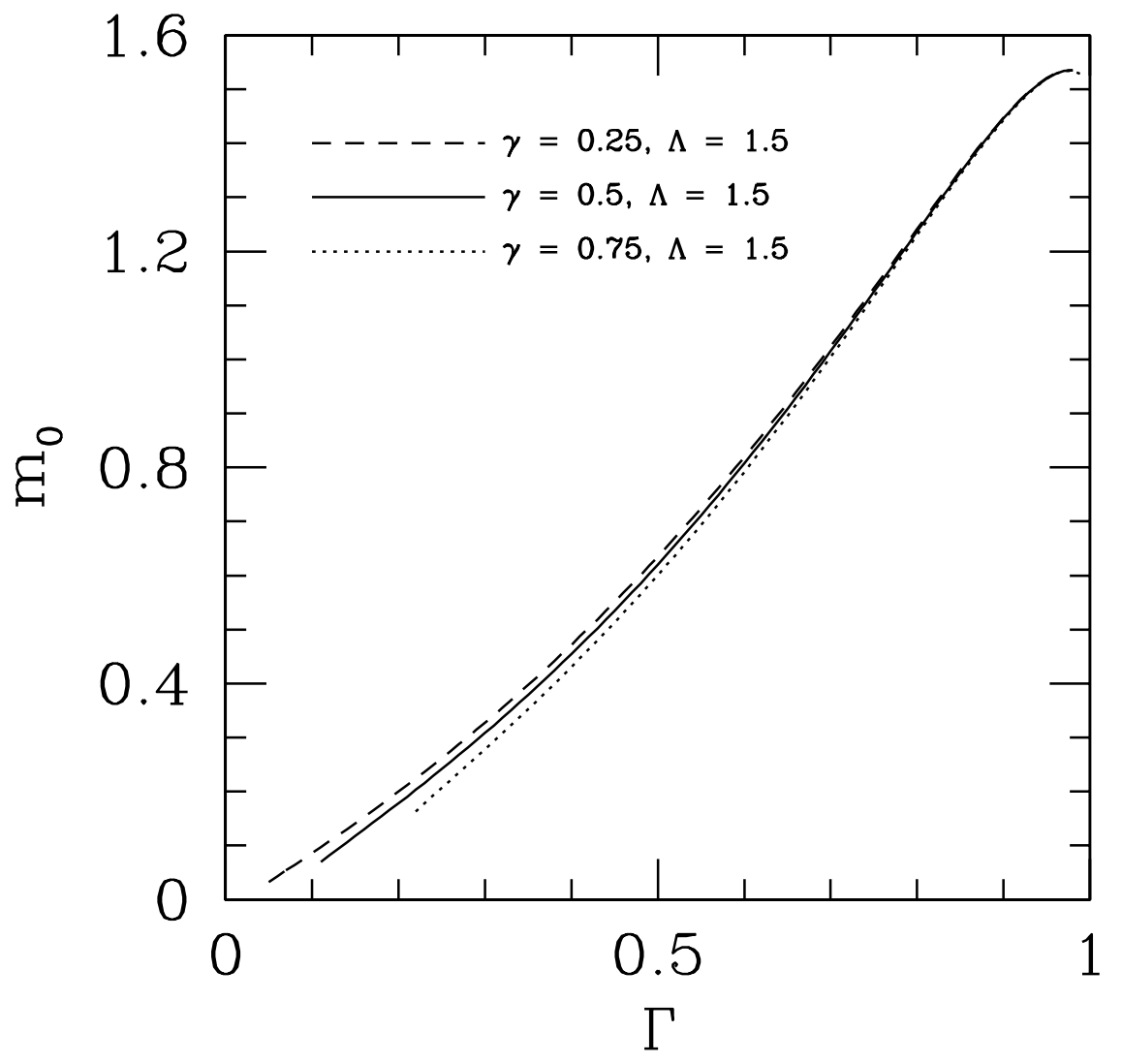

Fig. 8. - The value of the reduced mass at the origin $m_{0}$ as a function of the static equation of state index $\Gamma$ for $\Lambda=1.5$ and three different values of the dynamic equation of state index $\gamma$. 


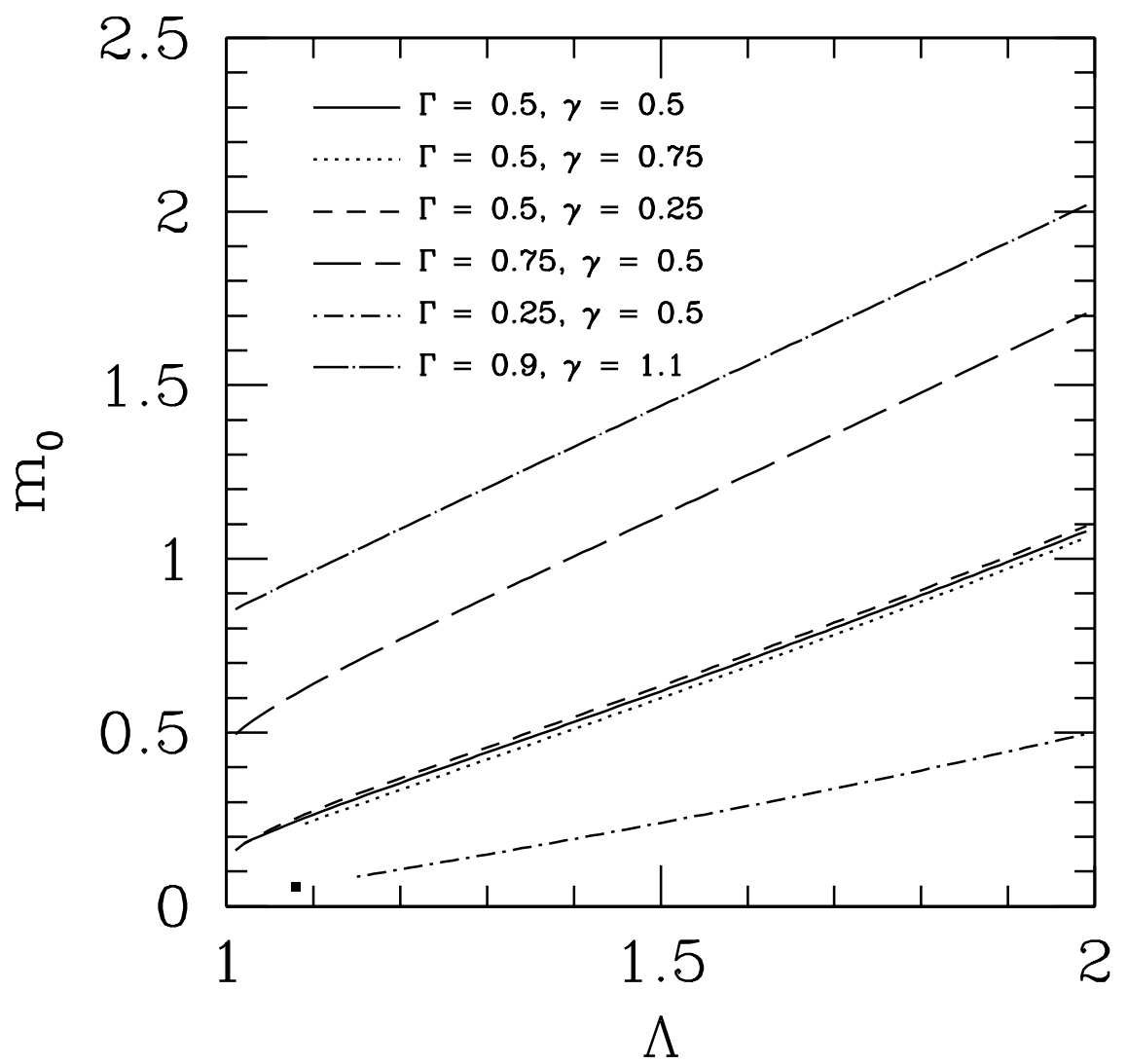

Fig. 9.- The value of the reduced mass at the origin $m_{0}$ as a function of the overdensity parameter $\Lambda=1.5$ for the specified values of the equation and state indices $\Gamma$ and $\gamma$. The solid square data point corresponds to the results obtained for a solution which passes smoothly through the singular surface, as discussed in $§ 5.1$. 


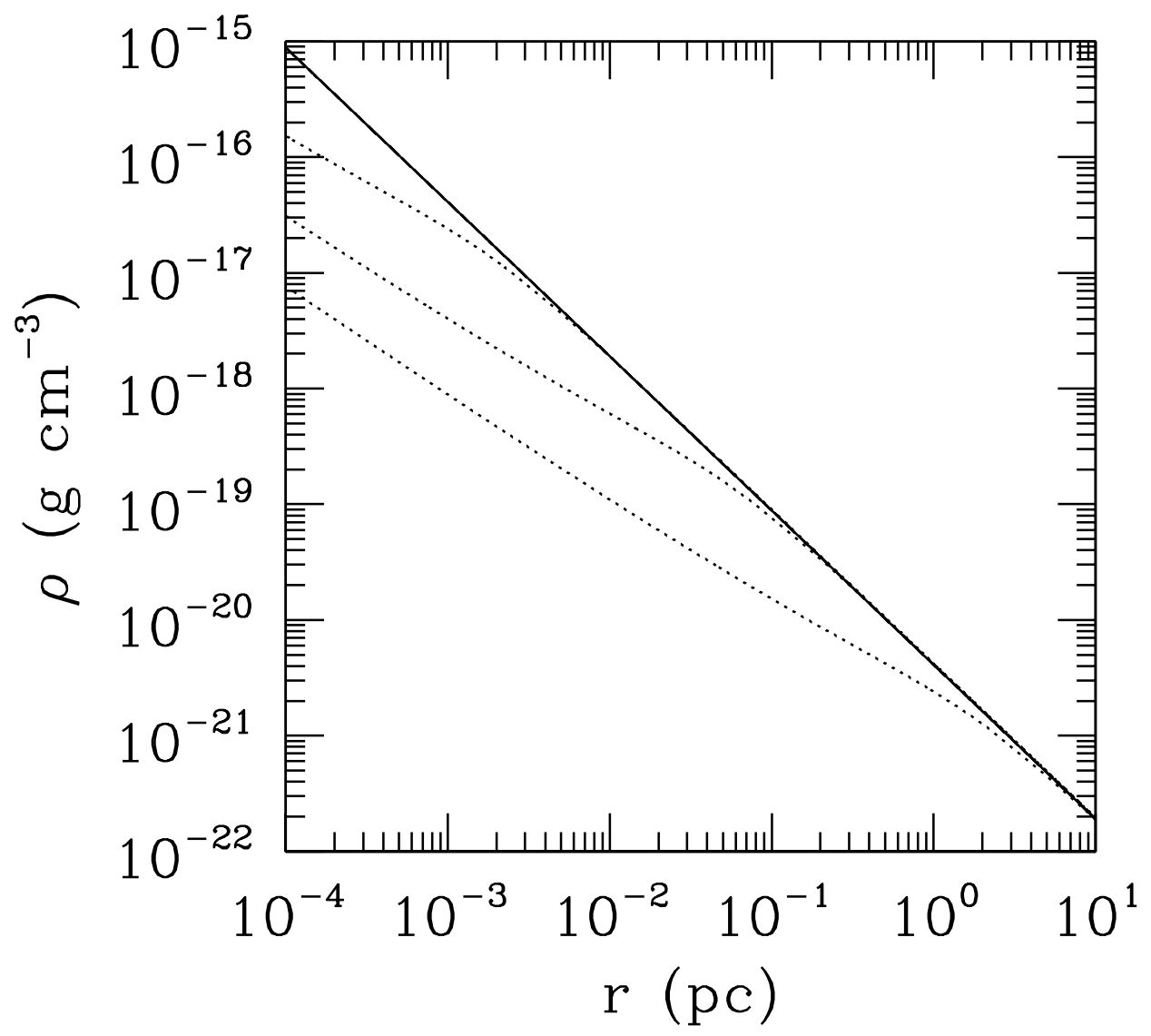

Fig. 10.- Density profiles illustrating the inside-out collapse of a cylindrical cloud initially at rest and overdense by a factor $\Lambda=1.5$, and for which $\Gamma=\gamma=0.5$. The solid curve represents the initial $(t=0)$ density profile $\rho=\Lambda \rho_{E}$, and the dotted curves show the profiles (from top to bottom) at times $t=10^{4}, t=10^{5}$ and $t=10^{6}$ years. 


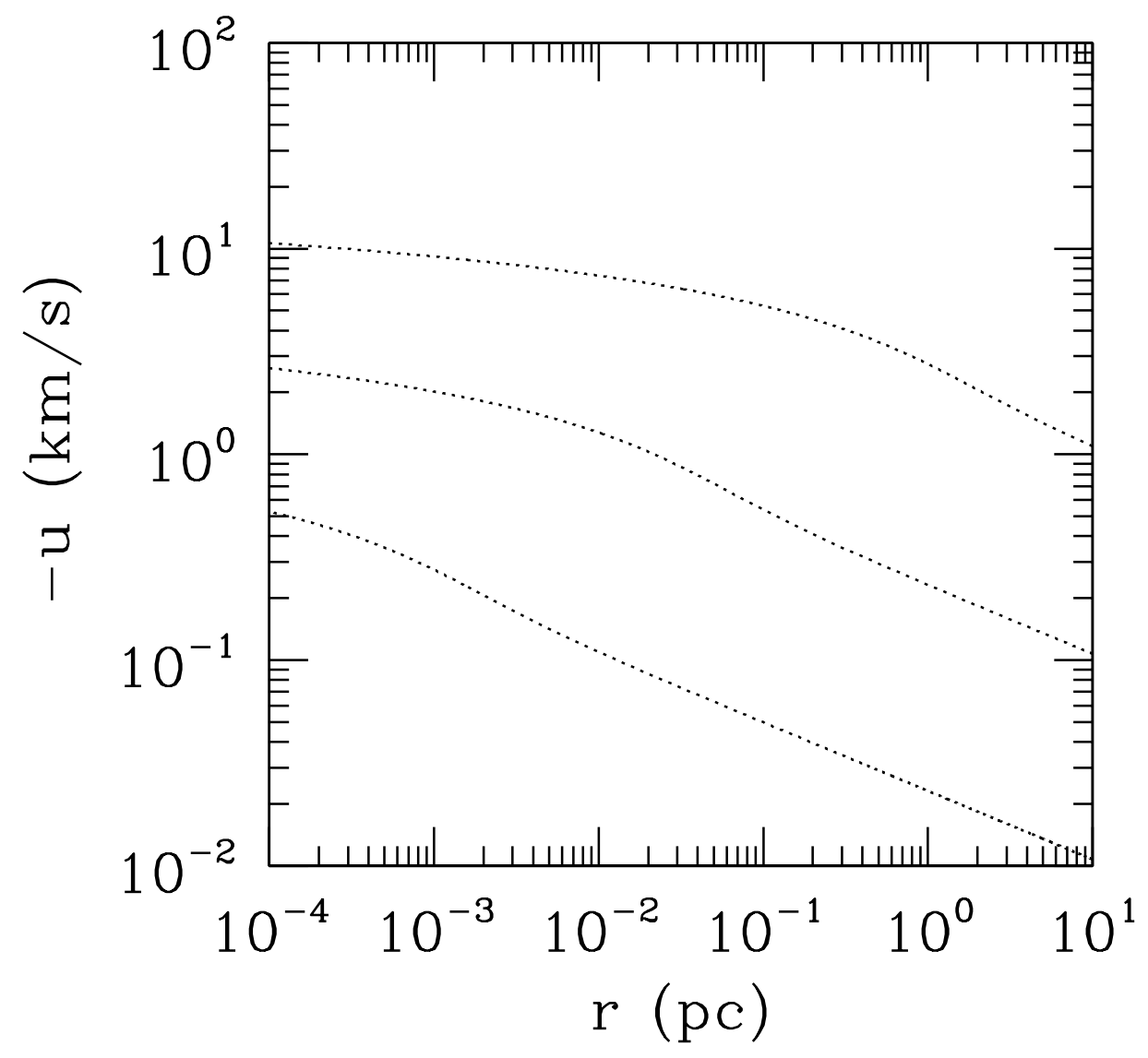

Fig. 11.- Velocity profiles illustrating the inside-out collapse of a cylindrical cloud initially at rest and overdense by a factor $\Lambda=1.5$, and for which $\Gamma=\gamma=0.5$. The dotted curves show the profiles (from bottom to top) at times $t=10^{4}, t=10^{5}$ and $t=10^{6}$ years. 


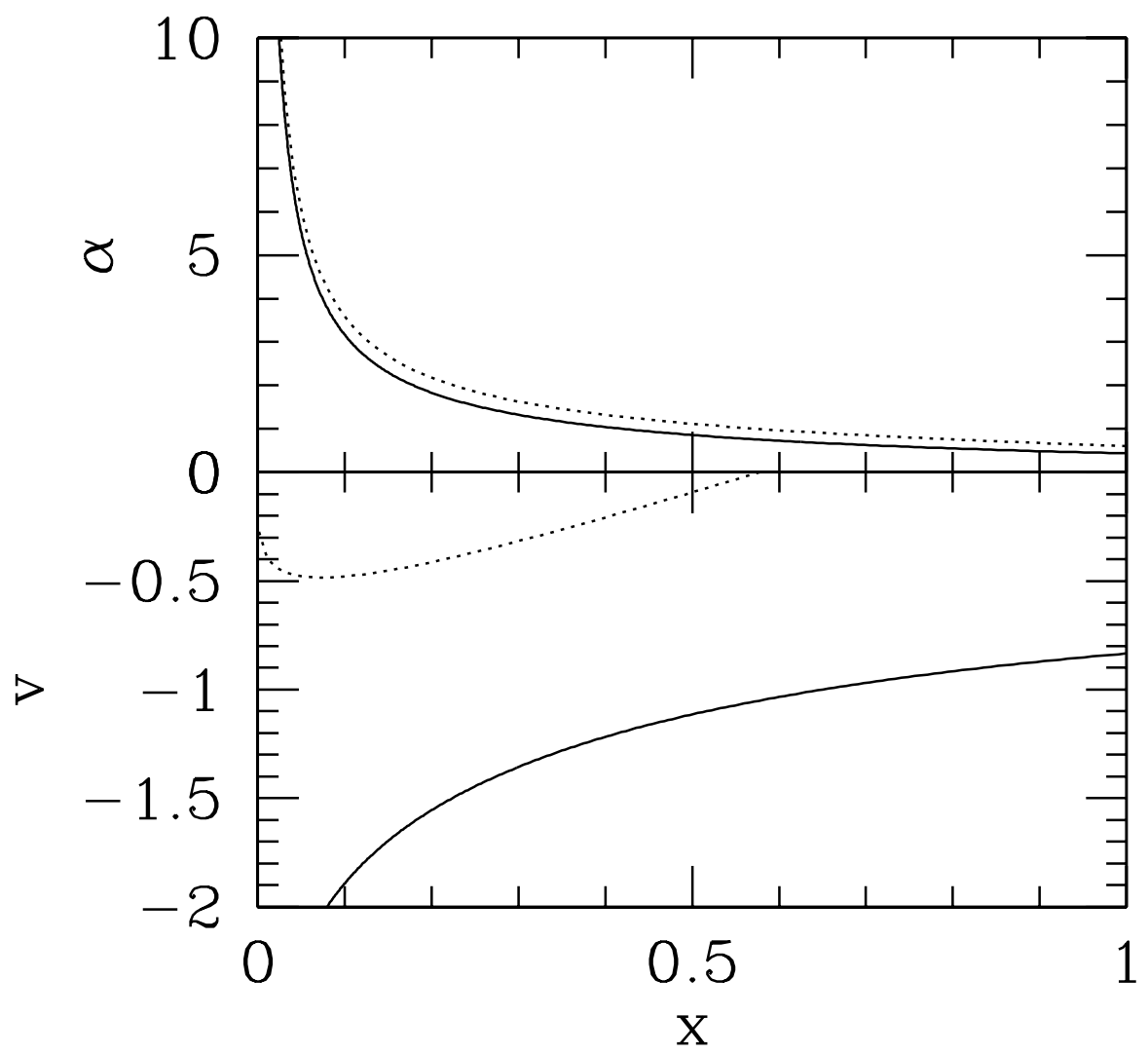

Fig. 12.- The dotted curves denote the density (upper panel) and velocity (lower panel) projections of the critical curve for the parameters $\Gamma=\gamma=0.5$ and $\Lambda=1.5$. The solid curve in each panel denotes the corresponding self-similar solution, also shown in Figs. 4 and 5. This solution does not pass through the critical curve. 


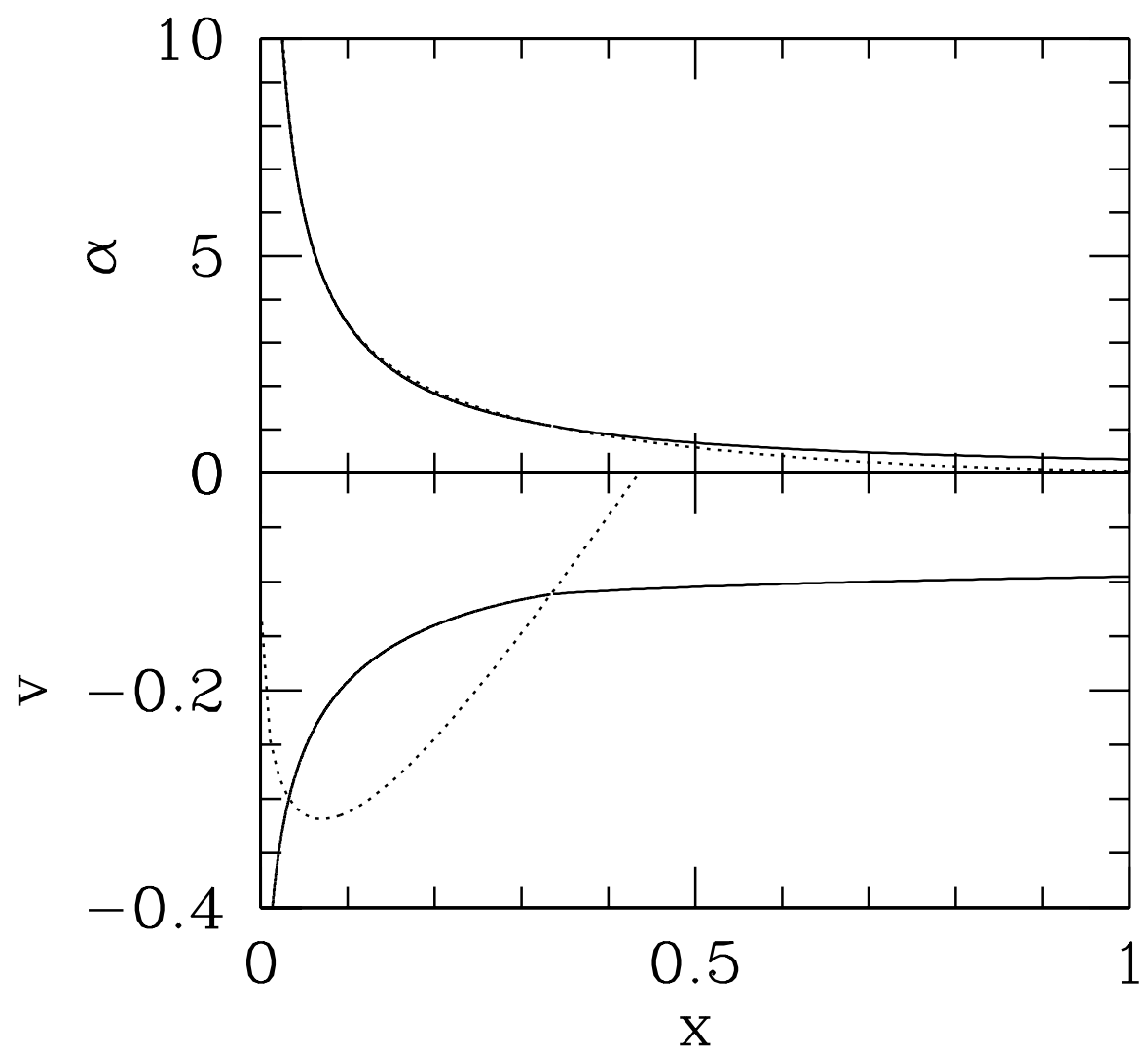

Fig. 13. - The dotted curves denote the density (upper panel) and velocity (lower panel) projections of the critical curve for the parameters $\Gamma=0.25, \gamma=0.5$, and $\Lambda=1.08$. The solid curve in each panel denotes the corresponding self-similar solution, which passes through the critical curve at $x_{c}=0.336$ and at $x_{c}=0.0322$. 


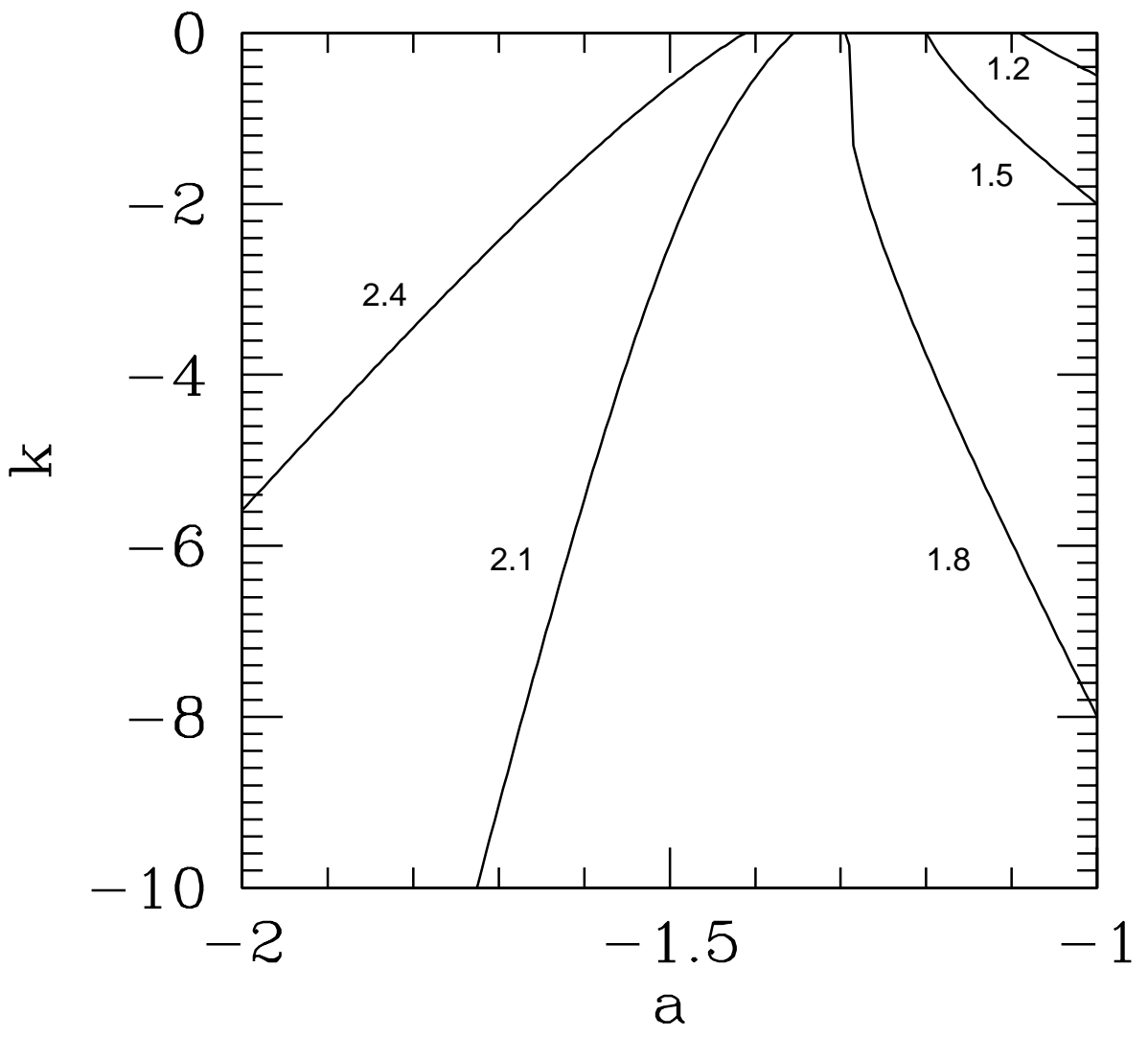

Fig. 14. - The negative (real) roots of Eq. $\operatorname{xxxxxx}$ as a function of $a$ for values of $C_{1}=$ $1.2,1.5,1.8,2.1$ and 2.4, as denoted in the figure. 


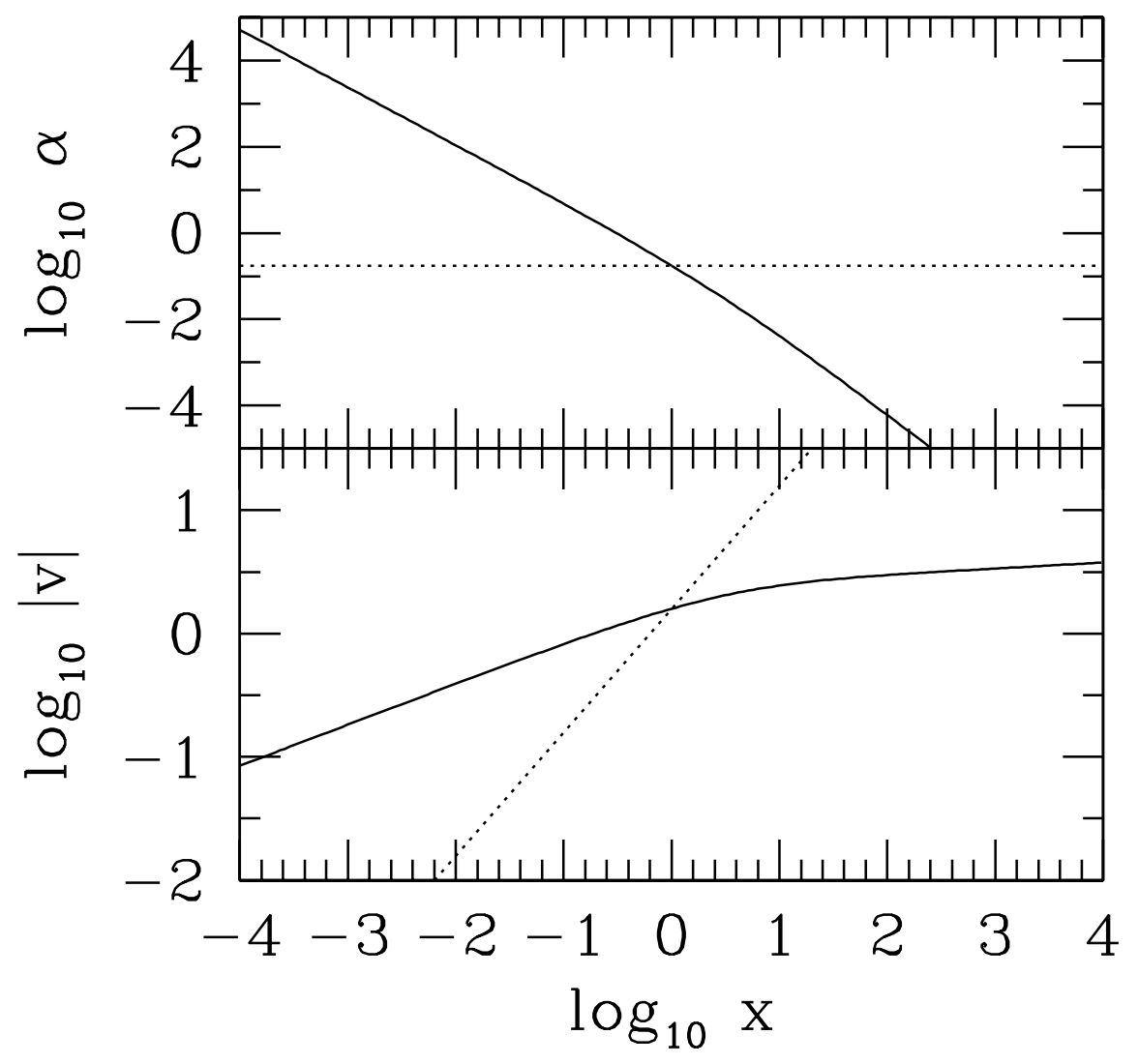

Fig. 15. - The dotted curves denote the density (upper panel) and velocity (lower panel) projections of the critical line for the parameters $a=-1.05, C_{1}=1.5$. The solid curve in each panel denotes the corresponding self-similar solution which passes through the critical line at the point $x_{c}=1$. 


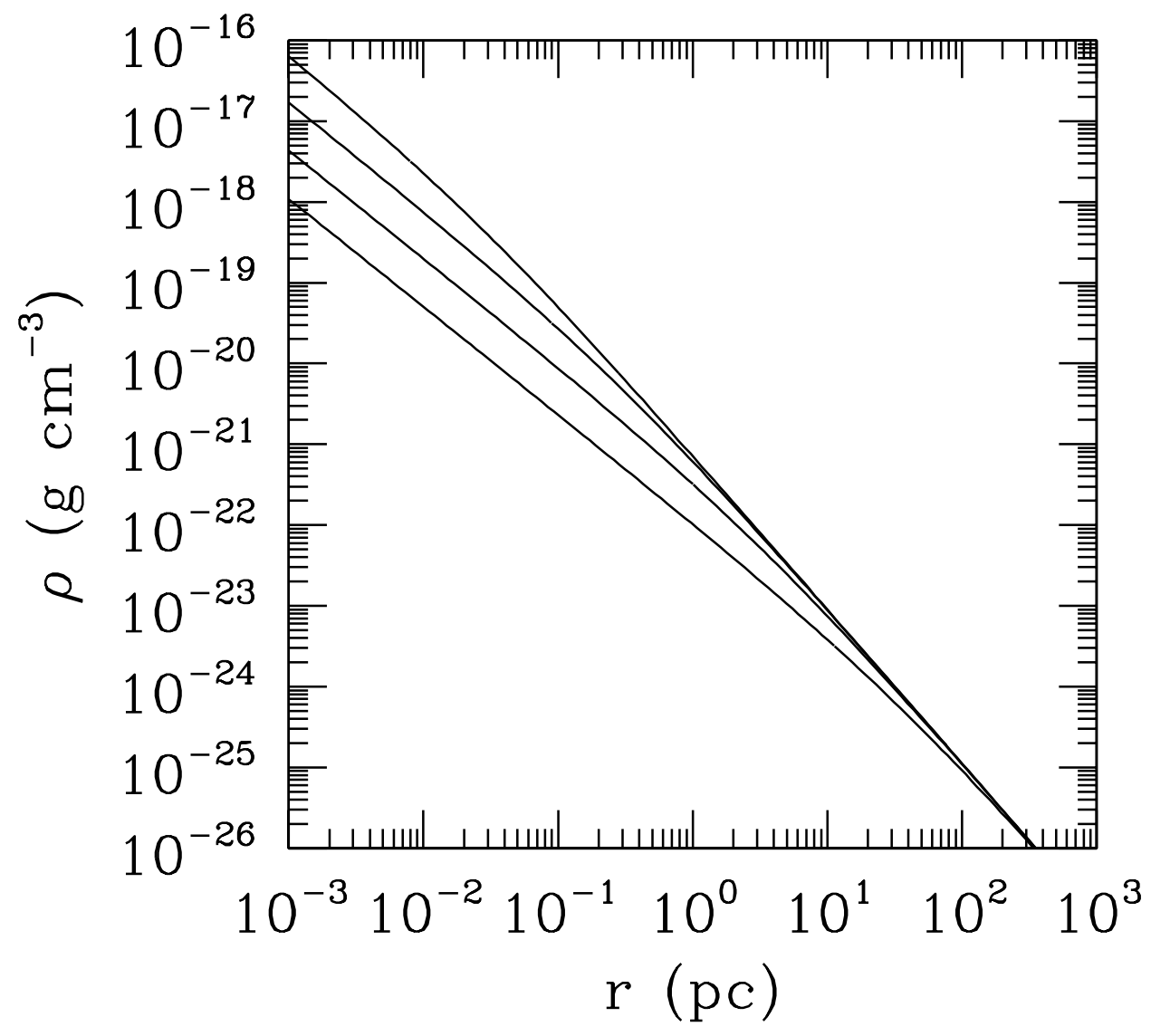

Fig. 16.- Density profiles associated with the self-similar solutions presented in Fig. 15, scaled by setting $x_{c}=1$ when $r=1 \mathrm{pc}$ and $t=1 \mathrm{Myr}$. The solid curves show the profiles (from top to bottom) at times $t=0.01,0.1,1$ and 10 Myrs. 


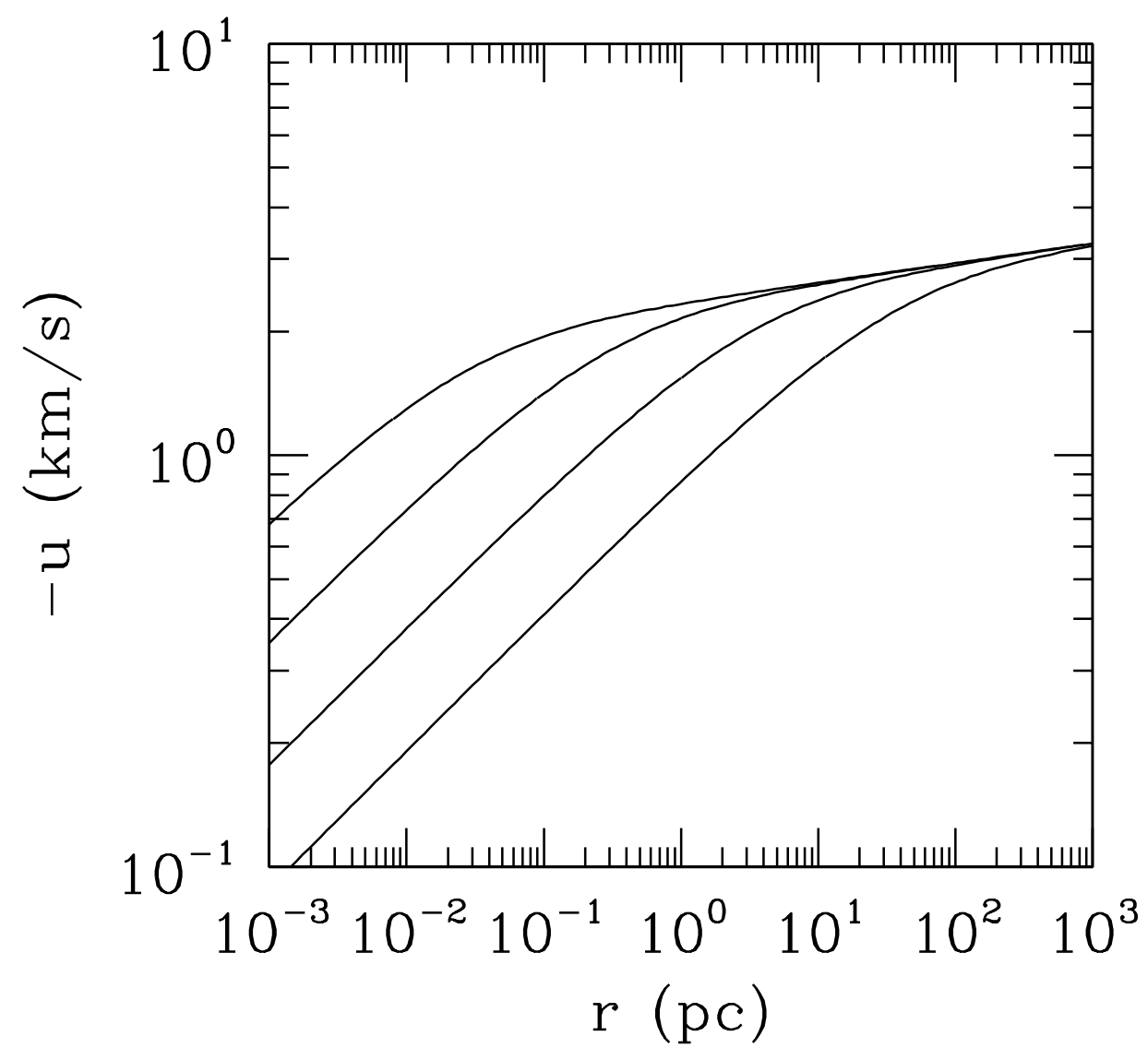

Fig. 17.- Velocity profiles associated with the self-similar solutions presented in Fig. 15, scaled by setting $x_{c}=1$ when $r=1 \mathrm{pc}$ and $t=1 \mathrm{Myr}$. The solid curves show the profiles (from top to bottom) at times $t=0.01,0.1,1$ and 10 Myrs. 